\title{
Gradability across grammatical domains
}

\author{
Margit Bowler and John Gluckman \\ University of Manchester | University of Kansas
}

This paper argues that ordered abstract scales are applicable to analyses of a range of grammatical domains. We argue this based on data from a number of unrelated languages, primarily Logoori (Bantu, JE 41; Kenya). The Logoori verb kudoka can be translated into English as 'to arrive'/ to reach,' 'to be enough,' and 'must,' depending on its linguistic context. We propose that these meanings arise from a single semantic denotation that is sensitive to a shared gradable component in the semantics of linguistic expressions referring to spatial paths, gradable predicates, measures of plural count nouns/mass nouns, and modals. The central theoretical issue addressed in this paper is the application of ordered, abstract scales in a model of grammar. This data and proposal is an important contribution to the literature arguing for a gradable model of modality (Lassiter 2014, 2017b, among others).

Keywords: semantics, gradability, gradable predicates, modality, Bantu

\section{Introduction}

This paper addresses the cross-categorial applicability of abstract scales in a model of semantics. We show that similar kinds of scales can be used to model the meanings of gradable predicates, spatial expressions, cardinalities of plural count nouns/sizes of portions of mass nouns, and modals. We explore this through an analysis of a recurring polysemy that is found in a range of unrelated languages.

In several languages, we find that a single lexical item can be used to express the meanings 'to reach/arrive, 'to be enough,' and some modal meaning, most frequently necessity modality. We illustrate this polysemy in (1) with examples from Logoori (Bantu, JE 41; ISO rag). The Logoori verb kudoka has a number of superficially distinct interpretations. In (1a), kudoka is translated as 'to arrive'/'to reach.' In (1b) and (1c), it is translated as 'to be enough.' And in (1d), it is translated as 'must.' We call these spatial, degree, amount, and modal uses, respectively. 
(1) a. Sira a-dok-i (Nairobi)

1Sira 1sM-DOK-FV Nairobi

'Sira arrived (at Nairobi).'

SPATIAL USE

b. li-boksi li-v-i na vu-angu vu-dok-an-a

5NC-box 5SM-COP-FV with 14NC-lightness 14sM-DOK-PL-FV

(ku-geng-w-a)

15NC-lift-PASS-FV

'The box is light enough (to be lifted).'

DEGREE USE

c. Sira a-v-i na ma-voyo ga-dok-an-a (ku-romb-a i-chai)

1Sira 1sM-COP-FV with 6NC-egg 6SM-DOK-PL-FV 15NC-make-FV 9NC-tea

'Sira has enough eggs (to make breakfast).'

AMOUNT USE

d. (ku ma-ragoo) ku-dok-a Sira a-zi-e yengo

to 6NC-law 15NC-DOK-Fv 1Sira 1sM-go-sBJv home

'(According to the law), Sira must go home.'

MODAL USE

A similar polysemy is found in Mandarin Chinese (Sino-Tibetan) with the lexical item gou. Gou has spatial, degree, and modal uses, as shown in (2) (Shuzhen Jiang, p.c.).

(2) a. Xiao-zhang shen shou qu gou na ben shu

Xiao-zhang stretch hand go GOU that CL book

'Xiaozhang stretched out to reach for that book.'

SPATIAL USE

b. zhe-xie shui-guo gou le, bu yong zai maile

these fruit GOU P NEG need again buy $P$

'These fruits are enough, and there is no need to buy more.' DEGREE USE

c. Xiao-zhang neng-gou zi-ji you-yong le

Xiao-zhang able-Gou self swim $P$

'Xiaozhang is able to swim by himself.'

MODAL USE

In Tatar (Turkic), the verb citergä has both spatial and amount meanings, as shown in (3). ${ }^{2}$

(3) a. xat irtägä kil-ep cit-eçek- $\varnothing$

letter tomorrow come-IP CIT-PROSP-3SG

'The letter will arrive tomorrow.'

SPATIAL USE

1. The modal use of gou, unlike kudoka, expresses modal possibility rather than necessity. However, this nonetheless supports positing a relationship between the spatial, degree, and modal meanings. We briefly discuss in $\S \mathbf{4 . 2 . 4}$ how this possibility meaning can be accounted for under our analysis.

2. The Tatar data is from the first author's fieldwork. 
b. bu qader su cit-eçek- $\varnothing$

this much water CIT-PROSP-3sG

'This much water will be enough.'

AMOUNT USE

In Chickasaw (Muskogean), the verb ona also has both spatial and amount meanings, as in (4) (Pam Munro, p.c.; Munro and Willmond 1995).

(4) a. Yamm-a ona-tok.

that-ACC ONA-PT

'He got there.'

SPATIAL USE

b. Pam oka' lawa-kat kaa isht=achif-a'ni-ka ona-ho ishko.

Pam water be.a.lot-CMP.ss car INST=wash-can-CMP.DS ONA-FOC.DS drink

'Pam drank enough water to wash a car.'

AMOUNT USE

We also find other languages in the Luhia subfamily of Bantu that have similarly polysemous lexical items. These lexical items are unrelated to Logoori kudoka. In (5), we show examples of the spatial and modal uses of the Lusaamia verb khwola. ${ }^{3}$ (We discuss what diachronic conclusions we can draw from the cross-Luhia data in $\S 6$.

(5) a. nda-ol-a

1SG-arrive-FV

'I arrived'.

SPATIAL USE

b. o-khw-ola o-fwal-e i-helmet

15AUG-15NC-must 2sG-wear-FV 9NC-helmet

'You must wear a helmet.'

MODAL USE

Finally, there is an unrelated lexical item in Logoori, the preposition mpaka, that shows the same polysemy as kudoka. ${ }^{4}$ Mpaka has similar spatial, degree, and modal uses, as shown in (6).

(6) a. Sira a-zi-i mpaka Nairobi

1Sira 1sm-go-FV MPAKa Nairobi

'Sira went until/up to Nairobi'.

SPATIAL USE

3. The Lusaamia data is from the second author's fieldwork.

4. Mpaka is borrowed from Swahili. In Swahili, it has a prepositional meaning ('until') and a nominal meaning ('border'). We have anecdotal evidence that mpaka has the same three-way polysemy in Swahili as well, though the modal necessity meaning is not accepted by all Swahili speakers, and for those speakers that accept it, it is acknowledged as colloquial. 
b. kitabu ni ki-doto sana mpaka vuri msomi a-nyal-a 7book COP 7-easy very MPAKA every 1student 1sM-can-FV ku-ki-som-a

\section{5-7om-read-FV}

'This book is easy enough that every student can read it.'

'The book is so easy, every student can read it.'

DEGREE USE

c. mpaka Sira a-zi-e Nairobi

MPAKA 1Sira 1sm-go-sBJV Nairobi

'Sira must go to Nairobi.'

MODAL USE

The theoretical takeaway from this data is that these superficially distinct meanings must share some semantic commonality, since the same polysemy is observed across a range of languages. In this paper, we focus on Logoori kudoka. We provide an analysis that reflects what we argue is the shared property of all the uses of kudoka in (1), namely, that they can be modeled using abstract, totally ordered scales. This analysis follows a long tradition of modeling gradable predicates as scalar (Cresswell 1976, Kennedy 1999, among others). This links scalar analyses of gradable predicates with proposals to treat spatial paths as sets of locations (Hohaus 2020, 2018), as well as proposals for measure operators that apply to nouns and yield values on the scale of real numbers (Rett 2014, Cresswell 1976). More controversially, this data provides empirical support for a gradable model of modality, and suggests that the kind of scale associated with modality shares some properties with the familiar degree scale used to model gradable predicates (Lassiter $2017 \mathrm{~b}$, among others). At the very least, this data suggest that languages can treat all of these types of scales similarly, and demonstrates the cross-categorial applicability of abstract scales in our model of grammar.

Informally speaking, our analysis is as follows: the spatial use of kudoka in (1a) involves a scale of ordered locations on a path. The degree use of kudoka in (1b) involves a gradable property scale of ordered degrees of height. The amount use of kudoka in (1c) involves a scale of cardinality of plural nouns (or sizes of portions of mass nouns). The modal use of kudoka in (1d) involves a scale of worlds ordered according to e.g. their goodness or likelihood. In all four uses, kudoka relates a value on one of these scales to a contextually supplied "threshold" value that must be met or exceeded. We provide our Logoori data in $\S 2$, and give our analysis in $\S 4$. We provide an alternate analysis that still makes reference to gradability in $\S 5 . \S 6$ concludes. For an abridged version of our analysis, see Bowler and Gluckman (2020). 


\section{Logoori data}

We give a brief overview of Logoori grammar in $\S 2.1$. We describe the degree use of kudoka in $\S 2.2$, the amount use in $\S 2.3$, the spatial use in $\S 2.4$, and the modal use in $\S 2.5$. All four uses of $k u d o k a$ share a gradable core.

\subsection{Background on Logoori}

Logoori is a Bantu language in the Luhia subfamily. It is spoken primarily in Western Kenya, with additional speaker groups in Tanzania and Uganda. It is spoken by approximately 600,000 people, and has at least two major dialectal distinctions. ${ }^{5}$ The judgments reported in this paper are all from one dialect.

Logoori has some fairly standard Bantu properties, including a strict SVO word order, heavy pro-drop, and wh-in-situ. Logoori also has an extensive noun class system encoded as noun class prefixes, subject markers, object markers, and adjectival concord, as demonstrated in Table 1.

Logoori verbs host agglutinative morphology encoding subject agreement, tense, aspect, and mood; they can also take derivational morphology, such as valency increasing and decreasing morphemes. We refer the reader to Nurse and Philippson (2003) for additional discussion of Bantu syntax and morphology.

\subsection{Degree use of kudoka ('to be enough')}

We begin this section with a brief discussion of some Logoori property concept lexemes (PCLs). ${ }^{6}$ We use the term "PCL" to refer to items that lexicalize meanings that are expressed by adjectives in languages that have adjectives (e.g. the English adjectives tall, short, good, bad, jealous, happy, etc.). Logoori has nominal, verbal, and adjectival PCLs. Nominal PCL predications in Logoori have the form "be with PCL"? This PCL predication strategy occurs in a range of unrelated languages

5. Logoori is anecdotally reported to have a "northern" and "southern" dialect. The differences between the dialects is not well understood at this point, but do not appear to be relevant for the facts discussed in this paper.

6. The term "property concept" was coined by Dixon (1982), who described seven classes of property concepts that are typically lexicalized as adjectives in languages that have adjectives. We use the term "property concept lexeme" following Francez and Koontz-Garboden (2015, 2017). In languages lacking adjectives, PCLs can be nouns or verbs. This term is therefore lexical category-neutral.

7. These "be with N" constructions are also used to express possession of referential, non-PCL nouns. 
Table 1. Noun classes in Logoori

\begin{tabular}{lllll}
\hline Class & Prefix $^{*}$ & Subject Agreement & Concord & Example \\
\hline 1 & $m(u)-$ & $y-/ a-$ & $m(u)-$ & msomi 'student' \\
2 & $v a-$ & $v a-$ & $v a-$ & vasomi 'students' \\
3 & $m u-$ & $g u-$ & $g u-$ & musaala 'tree' \\
4 & $m i-$ & $j i-$ & $j i-$ & misaala 'trees' \\
5 & $l i-$ & $l i-$ & $l i-$ & libarabandi 'loquat' \\
6 & $m a-$ & $g a-$ & $g a-$ & mabarabandi 'loquats' \\
7 & $k i-$ & $k i-$ & $k i-$ & kitabu 'book' \\
8 & $v i-$ & $v i-$ & $v i-$ & vitabu 'books' \\
9 & $i-$ & $e-$ & $i-$ & enyumba 'house' \\
10 & $z i-$ & $z i(n)-$ & $z i-$ & zinyumba 'houses' \\
11 & $l u-$ & $l u-$ & $l u-$ & lugano 'rock' \\
12 & $k a-$ & $k a-$ & $k a-$ & kanyumba 'little house' \\
13 & $t u-$ & $t u-$ & $t u-$ & tunyumba 'little houses' \\
14 & $v u-$ & $v u-$ & $v u-$ & vutambe 'height' \\
15 & $k u-$ & $k u-$ & $k u-$ & kuzia 'to go/going' \\
21 & $g u-$ & $g u-$ & $j i-$ & gunyumba 'big house' \\
22 & $j i-$ & $j i-$ & jinyumba 'big houses' \\
\hline & & &
\end{tabular}

* Class 11 forms plurals in class 10, e.g., zingano.

** Logoori noun class prefixes can optionally occur with a preceding vowel called an augment (also called a pre-prefix). The function and distribution of the augment is not well-understood in Logoori, and in fact varies greatly across Bantu languages (Halpert to appear).

around the world; Francez and Koontz-Garboden $(2015,2017)$ term it "possessive predication."

(7) Sira a-v-i na vu-tambe.

1Sira 1sM-COP-FV with $14 \mathrm{NC}$-tallness

'Sira is tall.'

(Lit. 'Sira is with tallness.')

(i) Sira a-v-i na mu-doga

1Sira 1sM-COP-FV with 3NC-car

'Sira has a car.' (Lit. 'Sira is with a car.') 
(8) a-v-i na zi-soni.

1SM-COP-FV with 10NC-shame

'S/he is ashamed.'

(Lit. 'S/he is with shame.')

(9) Sira a-v-e na vu-ti

1Sira 1sM-COP-FV with 14NC-shyness

'Sira is shy'

(Lit. 'Sira is with shyness.')

In its degree use, kudoka occurs inside a subject relative clause that is headed by the nominal PCL. ${ }^{8}$ This relationship is reflected by noun class agreement between kudoka and the PCL. In (10), the class 14 nominal PCL vutambe 'tallness' triggers noun class 14 agreement ( $v u$-) on $k u$ doka. ${ }^{9}$ A literal translation for (10) could be 'Sira is with a height that reaches.' Thus, in its degree use, kudoka is unambiguously a verb. We provide an example of a Logoori subject relative clause without kudoka in (11) for comparison.

(10) Sira a-v-i na vu-tambe vu-dok-an-a

1Sira 1sM-COP-FV with 14NC-tallness 14SM-DOK-PL-FV

'Sira is tall enough'.

8. Degree uses of kudoka are only available in combination with nominal PCL predicates. Logoori speakers can use the lexical item sam (or samu) to express 'to be enough' with verbal, adjectival, and nominal PCL predicates; -angu 'light' in (i) is an adjective. Sam lacks the spatial and modal polysemies of kudoka; sam-expressions also have an associated value judgment that the degree of the gradable predicate is pleasant or good. Compare (i) to (12).

(i) li-boksi ni li-angu \{samu / *vu-dok-an-a / ${ }^{\star}$ ku-dok-a / $\left.{ }^{\star} l i-d u k-a n-a\right\}$ 5NC-box COP 5sM-light SAM / 14sM-DOK-PL-FV / 15NC-DOK-FV / 5SM-DOK-PL-FV ku-geng-a

$15 \mathrm{NC}-$ lift-FV

'The box is light enough to lift.'

'The box is just the right weight to lift.'

We argue that sam differs from kudoka in that (i) it has a much wider distribution and can combine with verbs and adjectives; and (ii) it contributes a value judgment.

9. A puzzling piece of morphology is the presence of -an in the degree (and amount) uses. Across Bantu languages the suffix - an has a number of diverse functions. It most widely marks reciprocity, but within a language may also mark (i) detransitivization; (ii) event plurality; (iii) individual (cumulative) plurality; (iv) reflexivity (Maslova 2003; Bostoen et al. 2015; Dom et al. 2018). In Logoori, it functions as an event pluralizer in other contexts (Gluckman 2018). Its presence in degree and amount uses of kudoka is a puzzle, which we do not have an answer to at present. It may be related to the fact that - an is also well known to appear in stative constructions (Seidl and Dimitriadis 2003). 
(11) o-mu-ndo a-v-i na i-nzara a-ri-i ma-ndazi 1AUG-NC-man 1sM-be-FV with 9NC-hunger 1sM-eat-FV 6NC-mandazi 'The man who was hungry ate the mandazi.'

An optional infinitival clause can further specify the degree of height, temperature, etc. that must be reached to count as "enough."

(12) li-boksi li-v-i na vu-angu vu-dok-an-a ku-geng-w-a

5NC-box 5SM-COP-FV with 14NC-lightness 14sM-DOK-PL-FV 15NC-lift-PASS-FV

'The box is light enough to be lifted.'

(13) i-nyingu i-v-i na vu-ritu vu-dok-an-a

9NC-pot 9sM-COP-FV with 14NC-heaviness 14NC-SM-DOK-PL-FV

ku-vunyany-a i-mesa

15NC-break-Fv 9NC-table

'The pot is heavy enough to break the table.'

(14) Sira a-v-i na vu-yanzi vu-dok-an-i ku-morom-a na

1Sira 1sM-COP-FV with 14NC-happiness 14SM-DOK-PL-FV 15NC-talk-FV with

Imali

IImali

'Sira is happy enough to talk to Imali.'

In the absence of this infinitival clause, there must be a threshold value that can be understood from the context.

\subsection{Amount use of kudoka ('to be enough')}

Amount uses of kudoka are syntactically identical to degree uses of kudoka. In both cases, kudoka occurs inside a relative clause that is headed by a noun. In amount uses of kudoka, this noun can be a plural count noun (e.g. mavoyo 'eggs') or a mass noun (e.g. amaaze 'water'). ${ }^{11}$ Kudoka again agrees with the noun class of this head noun.

The semantic contribution of kudoka in its amount use is the same as in its degree use. The contextual threshold value that must be reached to count as

10. Mandazi are fried dough balls commonly eaten throughout East Africa.

11. Count nouns in Logoori have different singular and plural forms that are marked by different noun class prefixes (livoyo/mavoyo 'egg/eggs'; imbwa/zimbwa 'dog/dogs'); see Table 1. However, mass nouns only occur with one noun class prefix (e.g. vuyanzi 'happiness', amaaze 'water'). Mass nouns generally occur in classes 6 or 14, but may appear in other classes as well (e.g. zisoni 'shame', in class 10). 
"enough" can be optionally specified by an infinitival clause, but if omitted it must be contextually given.

(15) Sira a-v-i na ma-voyo ga-dok-an-a (ku-romb-a i-chai) 1Sira 1SM-COP-FV with 6NC-egg 6sM-DOK-PL-FV 15NC-make-Fv 9NC-tea 'Sira has enough eggs (to make breakfast).'

(Lit. 'Sira is with eggs that reach (to make tea).')

(16) ku-v-i na a-ma-aze ga-dok-an-i (ku-menyel-a) 1PL-COP-FV with 6AUG-6NC-water 6SM-DOK-PL-FV 15NC-survive-FV 'We have enough water (to survive).'

(Lit. 'We are with water that reaches (to survive).')

(17) Sira a-gur-i zi-karama zi-dok-an-i ku-vogor-a li-gera 1Sira 1sM-buy-Fv 10NC-pencil 10SM-DOK-PL-Fv 15NC-take-FV 5NC-test 'Sira bought enough pencils to take the test.'

Kudoka is unacceptable in combination with singular count noun subjects. Examples like (18) are felicitous only if the singular count noun is coerced into a mass noun interpretation.

(18) *Sira a-v-i na li-voyo li-dok-an-a (ku-romb-a i-chai) 1 Sira 1sM-COP-FV with 5NC-egg 5SM-DOK-PL-Fv 15NC-make-FV 9NC-tea Intended: 'Sira has enough egg (to make breakfast).'

\subsection{Spatial use of kudoka ('to arrive'/'to reach')}

In its spatial use, kudoka is translated as 'to arrive' or 'to reach.' It is unambiguously a verb in its spatial use; it inflects for subject marking, tense, aspect, and mood. The noun encoding the goal of motion (i.e., the threshold) is optional. When it is omitted, the location of arrival must be understood through discourse, but it need not be "speaker-oriented," i.e., indexically associated with the location of the speaker.

(19) Sira a-dok-i (Nairobi)

1Sira 1sM-DOK-FV Nairobi

'Sira arrived (at Nairobi).'

'Sira reached (Nairobi).'

This optional goal noun is not a direct object of kudoka. It cannot be passivized (20a) or object marked (2ob). We include this data to show that the goal noun is syntactically equivalent to the other contextual thresholds in the degree, amount, and modal uses; that is, it is never a direct object of kudoka. 
(20) a. * Nairobi i-dok-w-i

9Nairobi 9sM-DOK-PASS-FV

Intended: 'Nairobi was reached.'

b. * Sira a-ki-dok-i

1Sira 1SM-9OM-DOK-FV

Intended: 'Sira reached it.'

\subsection{Modal use of kudoka ('must')}

In its modal use, kudoka typically occurs in its infinitival form. ${ }^{12}$ Its infinitival form is indicated by noun class 15 marking $(k u-)$ and the final vowel $-a$. Infinitival kudoka obligatorily embeds a subjunctive clause, which is diagnosed by the verbal suffix -e. Infinitival kudoka may occur either clause initially or directly after the subject. $^{13}$

(21) a. ku-dok-a Sira a-zi-e yeng'o

15NC-DOK-FV 1Sira 1sM-go-sBJv home

'Sira must go home.'

b. Sira ku-dok-a a-zi-e yeng'o

1Sira 15NC-DOK-FV 1sM-go-SBJV home

'Sira must go home.'

Modal uses of kudoka are compatible with all Kratzerian flavors of necessity modality (Kratzer 1981, 1991). ${ }^{14}$

12. Modal uses of kudoka can also occur in an "impersonal" form in which kudoka takes an event pluralizer (-an) (Gluckman 2018) and either noun class $6(\mathrm{ga})$ ) or noun class $9(e-)$ as an "expletive" subject agreement (Gluckman and Bowler 2016). We do not discuss this syntactic configuration in this paper, though our analysis extends straightforwardly to cover this data.

(i) ga-dok-an-a ndee a-va-rwaye va-v-e na ki-bande chi-a 6SM-DOK-PL-FV that 2AUG-NC-patient 2SM-COP-SBJV with 7NC-card 7AGR-ASSO

ki-vitale ku-lola mu-ahi

$7 \mathrm{NC}$-identity $15 \mathrm{NC}$-see $1 \mathrm{NC}$-doctor

'It's necessary that patients have an ID card to see a doctor.'

We note, however, that while (i) is demonstrably biclausal, the modal uses of kudoka in which it appears in the noun class 15 form (as in (21) et seq) are demonstrably not biclausal.

13. Bantu class 15 has well-known properties of both verbal and nominal syntax (Mugane 1997). We make the assumption that the modal use of kudoka has the category verb, rather than noun. We do not believe that this affects our eventual analysis.

14. The elicitation of modality was guided in part by Vander Klok (2014); see Gluckman and Bowler (2020) for a description of modality in Logoori. 
(22) Epistemic necessity: Kageha is in the library. She sees that the people who are coming into the library are carrying wet umbrellas, and that their clothes are wet. Kageha thinks:

ku-dok-a i-mbula i-v-i ne-i-kubang-a

15NC-DOK-FV 9NC-rain 9sM-COP-SBJV COMP-9sM-beat-FV

'It must be raining.'

(Lit. 'The rain must be beating.')

(23) Deontic necessity: In Kenya, the law states that when you ride a motor bike:

ku-dok-a w-evek-e i-gudwe

15NC-DOK-FV 2sG-wear-SBJV 9NC-helmet

'You must wear a helmet.'

(24) Teleological necessity: AFC Leopards (Ingwe) are playing in a tournament. In order to advance: ${ }^{15}$

ku-dok-a Ingwe va-leg-e Gor Mahia

15NC-DOK-Fv 9Ingwe 2sm-beat-Fv Gor Mahia

'Ingwe must beat Gor Mahia.'

(25) Circumstantial necessity: You are on a bus to Nairobi. You have not had a chance to go to the toilet for 6 hours, and your bladder is full. You text your friend:

ku-dok-a inz-inyaal-e

15NC-DOK-FV 1sG-urinate-SBJV

'I have to pee.'

The source of the necessity can be optionally overtly specified, as in the following deontic example.

(26) ku ma-ragoo ku-dok-a Sira a-zi-e yengo

to 6NC-law 15NC-DOK-FV 1Sira 1sm-go-sBJv home

'According to the law, Sira must go home.'

The degree and amount uses of kudoka both express something having to do with "sufficiency." One therefore might expect that the modal use of kudoka would express weak rather than strong necessity (i.e., 'should' rather than 'must'). This is not the case; Logoori uses the verb kwenya for weak necessity (see Gluckman and Bowler 2020 for much more information on the Logoori modal system).

15. Ingwe is the name of the football/soccer team from Kakamega county. It is primarily made up of Luhia, who have a friendly rivalry with the neighboring Luo represented by Gor Mahia. The word ingwe means 'leopard.' 


\section{Background on gradability}

The conceptual starting point of our analysis of kudoka is that all four of its uses described in $\S 2$ intuitively invoke some sort of gradable scale. By "scale," we refer to a set of items that are subject to an ordering; henceforth, when we say that something is "gradable," we mean that it can be modeled using such a scale. In the spatial use in $\S 2.4$, the scale is one of ordered locations on a path in space. In the degree use in $\S 2.2$, the scale is an ordered property scale, the dimension of which is lexically specified by the nominal PCL. In the amount use in $\S 2.3$, the scale is one of ordered cardinalities of plural count nouns/ordered sizes of portions of mass nouns. In $\S 2.5$, the scale is one of ordered possible worlds relative to what is "best" in a given context.

Since all four contexts that kudoka appears in share a gradable core, we propose an analysis that adapts concepts from the degree semantic literature, which has a long history of using scales to model meaning. Degree scales consisting of totally ordered sets of degrees were initially posited to model the behavior of gradable adjectives (Cresswell 1976, Bierwisch 1989, among many others). Since degrees scales are the most robustly studied kind of linguistic scales, we use the degree literature as a jumping-off point for the exposition of our analysis. In this section, we explain some of the background assumptions and formal tools used to model gradability. We address the applicability of abstract, ordered scales to other linguistic domains; namely, spatial descriptions, measures of plural count/mass nouns, and modals. We propose in $\S 4$ that $k u$ doka is sensitive to the property of gradability as it occurs across semantic types, i.e., in the domains of locations, measures of individuals, and worlds.

\subsection{Gradability and adjectives (PCLs)}

Degrees (of type $d$ ) were introduced into the semantic ontology to account for gradability in language. They were initially posited to model the behavior of gradable adjectives like big and tall (Cresswell 1976, Bierwisch 1989, Kennedy 1999, among many others). Gradable adjectives can combine with degree modifiers like very, which in (27) contributes that the degree of Leroy's height greatly exceeds some contextual standard degree of tallness. Gradable adjectives can also participate in comparison constructions; (28) asserts that the maximum degree of Leroy's height is strictly greater than the maximum degree of Susan's height. Both (27) and (28) refer to degrees on a scale of height/tallness.

(27) Leroy is very tall.

(28) Leroy is taller than Susan. 
Degree semantic analyses of gradable adjectives posit a downward monotonic scale consisting of a dense set of degrees that are totally ordered with respect to some dimension. ${ }^{16}$ That is, degree scales are treated as triples $\langle\mathrm{D},>, \delta\rangle$, where $\mathrm{D}$ is a set of degrees, $>$ is a total ordering of $\mathrm{D}$, and $\delta$ is the lexically specified dimension of the adjective (e.g. height in the case of tall/short; temperature in the case of hot/ cold). Being subject to a total ordering is therefore part of what it means to be a degree.

The degree scale associated with tall can be conceptualized as in $(29):{ }^{17}$ as an individual's degree on the scale of height increases, that individual's tallness increases. $^{18}$

scale of tall

$$
\begin{array}{llllllllll}
\dot{d}_{1} & d_{2} & \dot{d}_{3} & \dot{d}_{4} & \dot{d}_{5} & \dot{d}_{6} & \dot{d}_{7} & \dot{d}_{8} & \dot{d}_{9} & \dot{d}_{10}
\end{array}
$$

Degree analyses of gradable adjectives require a way to map the individual modified by a gradable adjective to a value on the lexically specified scale. This is typically accomplished via a measure function, which relates individuals to degrees. ${ }^{19}$ The semantics of an English gradable adjective like tall are therefore as in (30); tall asserts that the measure (degree) of $x$ 's height is a degree $d$. In positive, context-dependent expressions like Leroy is tall, the open degree argument of tall is assumed to be bound by a phonologically null operator that contributes that $d$

16. Downward monotonicity: If a degree $d$ is included in a set of degrees, $d-1$ is also included in that set. Density: For any two degrees $d, d^{\prime}$, there exists a degree $d^{\prime \prime}$ that occurs between them on the scale.

17. Kennedy and McNally (2005) show that (English) gradable adjectives vary in the kinds of degree scales that they are associated with. For example, positive assertions of minimumstandard adjectives like wet (bent, wrinkled) are true as long as the subject holds some nonzero degree of wetness, i.e., it exceeds a minimum standard degree. Positive assertions of maximumstandard adjectives like $d r y$ (straight, full) are true only if the subject holds a maximal degree of dryness. Our Logoori data suggests that Logoori PCLs can also lexicalize different kinds of scales; for instance, certain Logoori modifiers will only combine with PCLs of certain scale types. However, for the purpose of this paper, we do not discuss differences in scale type.

18. We follow Kennedy (2001) in assuming that antonyms involve reversing the order of the scale. Given a gradable antonym like short, an individual's shortness increases as their degree on a scale of height decreases.

19. This measure function can be encoded as part of the gradable adjective, which relates degrees to sets of individuals (Cresswell 1976, among many others; type $\langle d,\langle e, t\rangle\rangle)$. Bartsch and Vennemann (1972) and Kennedy (1999) take gradable adjectives themselves to denote measure functions from individuals to degrees (type $\langle e, d\rangle$ ). 
significantly exceeds the contextual standard degree of tallness (e.g. Pos; Kennedy 1999, 2011). ${ }^{20}$

(30) $\llbracket$ tall $\rrbracket=\lambda d \lambda x$. tall $(x, d)$

(where tall is a measure function that maps $x$ to a degree $d$ on a scale of height)

The use of scales like in (29) are well motivated and have a long history of application to gradable adjectives. We take the ability of Logoori PCLs to occur in comparatives, measure phrases, subcomparatives, and other expressions that are easily analyzed with degree semantics to motivate positing objects of type $d$ as part of the semantic toolkit of Logoori (von Stechow 1984; Beck et al. 2009).

(31) Sira a-v-i na vu-yanzi ku-vet-a Imali

1Sira 1sM-COP-GV with $14 \mathrm{NC}$-happiness $15 \mathrm{NC}-$ exceed-FV 1 Imali

'Sira is happier than Imali.'

\subsection{Gradability and plural count/mass nouns}

Plural count nouns (eggs, cats) and mass nouns (water, sand) can both be measured. Plural count nouns can be measured using numerals (six eggs, four cats). Mass nouns can be measured using units like gallon or cup, which can themselves combine with numerals (six gallons of water, four cups of sand).

Several authors model nominal measurements using tools from the degree literature (Cresswell 1976; Grosu and Landman 1998; Rett 2018, 2014). For instance, Rett (2014) follows Cresswell (1976) in proposing that quantities of count nouns can be measured using a measure function that combines with a plural individual and returns its cardinality. This cardinality is treated as being of type $d$. This (and other analyses) reflect the intuition that cardinality scales (consisting of the set of positive real numbers) share similarities with degree scales: like degree scales, cardinality scales are downward monotonic and totally ordered. ${ }^{21}$

We take the ability of kudoka to combine with plural count and mass nouns (as in its amount use in $\$ 2.3$ ) to reflect the presence of a measure operator like that proposed by Rett (2014)/Cresswell (1976). In the case of plural count nouns, the output of this measure operator is the cardinality of the plural individual

20. The context-dependence associated with gradable adjectives disappears in certain environments (e.g. in comparatives, with measure phrases) (Rett 2015). However, it is recognized as a property of positive expressions like Leroy is tall.

21. There is an extensive literature showing that plural count nouns and mass nouns should be modeled as having internal structure (Link 1983, among others). In $\S$ 4.2.2 we mediate between $k u d o k a$ and the denotation of the noun using a cardinality operator. 
denoted by the noun, represented as something of type $d$. In the case of mass nouns, the output is a measure of the sizes of portions of the noun, also treated as something of type $d$. (We spell out the precise semantics of these measure operators in $\S$ 4.2.2). In both cases, the output is a degree on a scale that stands in for the set of positive real numbers.

\subsection{Gradability and spatial paths}

Other authors have previously noted the semantic similarities and synchronic relationships between gradable adjectives and linguistic expressions referring to spatial paths or locations (Faller 1999; Winter 2005; Schwarzschild 2013, 2012; Hohaus 2020, 2018, 2012). Still other authors have modeled spatial paths as sequential (i.e., as gradable objects), but without reference to the literature on gradability in adjectives (Cresswell 1978, Krifka 1998). We take the Logoori data to show that languages can and do treat both PCLs and spatial expressions similarly in the grammar.

For our purposes, we model spatial paths as sets of locations. Following Hohaus (2020, 2018), we assume a semantic type $l$ for locations. We assume that, given a set of locations $L$, the locations in $L$ are ordered sequentially. $l_{n}$ precedes $l_{n+1}$ by virtue of its order in the sequence; the first location in the sequence is the starting point of the path, and the last location in the sequence is the end point of the path. ${ }^{22}$ Sets of locations (i.e., spatial paths) are therefore downward monotonic and totally ordered.

This treatment of spatial paths is a simplification of what paths look like in physical reality. Spatial paths can be circular, in that the start and end points of a path in space can be identical. The model we assume cannot capture this, since locations are inherently sequentially ordered. This model also does not account for the directionality of the path, and is restricted to change in one dimension. As a result, it does not capture the two-dimensional changes that differentiate a path that winds and doubles back on itself from a straight path. This model therefore treats the two paths in Figure 1 the same.

We are not troubled by the inability of our model to account for the differences between the paths in Figure 1; in fact, for the purposes of our analysis of kudoka, this is a virtue. Kudoka is not sensitive to two-dimensional change in paths. To demonstrate this, let the end points of both paths in Figure 1 be Nairobi.

22. Another way of accomplishing this would be to treat spatial paths as consisting of locationtime pairs. Since times are inherently totally ordered, location-time pairs are also totally ordered. This is akin to what Krifka (1998) and Cresswell (1978) propose in their analysis of paths. 


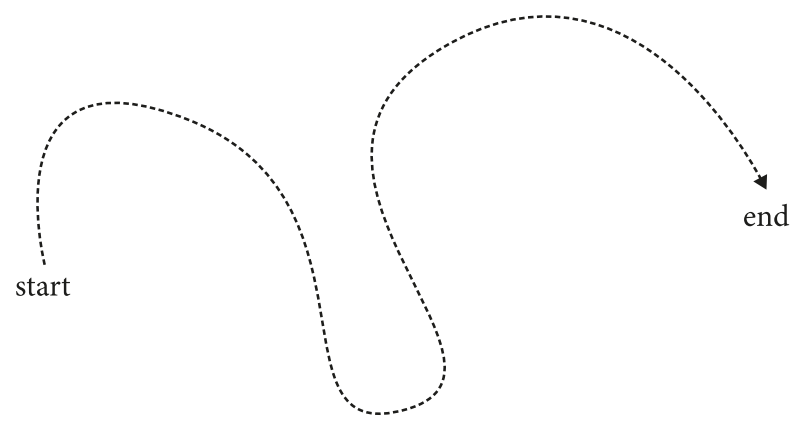

start end

Figure 1. A winding path and a straight path

If Sira reached Nairobi, it is felicitous to say (32) (repeated from $\$ 2.4$ ) regardless of whether Sira took the winding path or the straight path to get there.

(32) Sira a-dok-i Nairobi

1Sira 1sM-DOK-FV Nairobi

'Sira arrived at Nairobi.' 'Sira reached Nairobi.'

A spatial path can therefore be conceptualized as in (33); note the similarity to the degree scale in (29).

(33) spatial path

$\begin{array}{llllllllll}l_{1} & l_{2} & l_{3} & l_{4} & i_{5} & l_{6} & i_{7} & l_{8} & i_{9} & l_{10}\end{array}$

We provide the following analogy: while gradable adjectives can relate an individual to a degree on a property scale, spatial expressions can relate an individual to a location on a spatial path. ${ }^{23}$ Both property scales (sets of degrees) and spatial paths (sets of locations) can be treated as totally ordered.

\subsection{Gradability and modality}

Gradability in modality has been previously discussed by Lewis (1973), Kratzer (1981, 1991), Portner (2009), Klecha (2014), and Lassiter (2017b), among many others. For example, Kratzer $(1981,46)$ discusses "grades of possibility" with respect to the German noun Möglichkeit 'possibility' and auxiliary kann 'can.' In (34), Möglichkeit combines with the gradable modifier gering 'slight.' In (35),

23. In $\S 4$, we accomplish this formally by positing a location function $\Lambda$ that maps individuals to the set of their locations. 
kann occurs in a morphologically explicit comparative construction that compares degrees of likelihood (compare with the gradable adjective data in (28)). Kratzer uses this data to motivate an ordering on the relevant possible worlds according to how many of a set of propositions (the "ordering source") they satisfy.

(34) Es besteht aber immer noch eine geringe Möglichkeit, dass der there is however always still a slight possibility that the Kastenjakl der Mörder war.

Kastenjakl the murderer was

'There is, however, still a slight possibility that Kastenjakl was the murderer'.

(35) Der Gauzner-Michl kann eher der Mörder sein als der Kastenjakl. the Gauzner-Michl can rather the murderer be than the Kastenjakl. 'Gauzner-Michl is more likely to be the murderer than Kastenjakl.'

Lassiter (2010, 2011, 2014, 2017a; b) and Villalta (2008), among others, explicitly model modal meanings using abstract scales. Lassiter (2010), following Yalcin (2010), proposes a model of English gradable epistemic modals like possible and likely in which they are associated with a scale of numerical probabilities. For example, Lassiter (2010) argues that (36) is true iff the probability of Jorge winning the race strictly exceeds the probability of Sue winning the race. This is modeled using a function mapping propositions (i.e., sets of possible worlds) to real numbers between $o$ and 1 (similar to the measure function denoted by tall in (30) that maps individuals to a degree of height).

(36) It is more likely that Jorge will win the race than it is that Sue will win.

The proper analysis of gradable modality is still very much an open and active research topic (see Lassiter 2017b). For the purpose of this paper, what is important is that modals, like gradable adjectives and spatial expressions, can be modeled as gradable. We follow Lassiter $(2014,2017 \mathrm{~b})$ and Portner and Rubenstein (2016) in assuming that possible worlds can be totally ordered on a scale, akin to degrees on the scale associated with tall in (29). ${ }^{24}$ This total ordering of worlds must be derived, since even when ranked according to an ordering source (as in Kratzer 1991), worlds are only partially ordered: different worlds could have different propositions that are true in them that amount to the same degree on a scale of e.g. goodness, leaving open the possibility that multiple worlds could

24. Lassiter (2010) argues that distinctions in scale type (described as open versus closed scales by Kennedy and McNally 2005 in the context of gradable adjectives) are relevant to an analysis of gradable epistemic modals. Logoori kudoka is not sensitive to distinctions in scale type, so we do not discuss these differences in our paper. 
be equally good. Lassiter (2017b) derives a total ordering by mapping worlds to totally ordered values on scales, while Portner and Rubenstein (2016) propose that the utterance context imposes a total ordering on worlds. In $\S 4.2 .4$, we propose that the total ordering on worlds is contributed by the subjunctive marking on kudoka. For the purpose of this paper, it is not important to us how the total ordering is derived; what is crucial to our analysis is that sets of worlds can be treated in this way.

While the dimensions of property concept scales are lexically specified, dimensions of modal scales are contextually specified in English (reflecting Kratzer 1991, 1981's observation that modal flavor is determined by context). ${ }^{25}$ This modal scale can be one of how well the worlds conform with laws, with the speaker's wishes or expectations, with how closely the world matches the actual world, or so on. Modal scales can therefore be treated analogously to property concept scales as triples $\langle\mathrm{W},>, \delta\rangle$, where $\mathrm{W}$ is a set of possible worlds, $>$ is a total ordering of $\mathrm{W}$, and $\delta$ is the contextually specified dimension of the scale. We provide a conceptual representation of an ordered scale of worlds in (37).

$$
\text { scale of worlds }
$$

conforming to the law

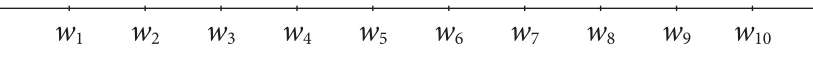

Assuming for example that the worlds in (37) are ordered based on laws, the higher the world is on the scale, the more laws are obeyed in that world. As a result, $w_{4}$ is "better" (i.e., higher-ranked) than $w_{3}$ because more laws are obeyed in $w_{4}$ than in $w_{3}$. In general, all modal expressions can be conceptualized in terms of "comparative goodness" or "comparative likelihood."

It is important to note that the Kratzerian model of possibility and necessity modals as existential versus universal quantifiers is not relevant to the gradable model of modality adopted here. Under a gradable analysis, possibility and necessity modals are defined with respect to the same scale, and do not differ in quantificational force. The difference between the two stems from the value on the scale that the proposition's value is compared to. To illustrate this, we can consider the examples Mary might be at home and Mary must be at home. The possibility expression is true iff the value assigned to the proposition "Mary is at home" meets or exceeds some point on a scale of likelihood. This point is lower for possibility modals than for necessity modals. Klecha (2014) argues that necessity modality is conceptually equivalent to superlativity - maintaining the

25. Gluckman and Bowler (2020) show that Logoori modals pattern like English in only lexically specifying force, and that flavor is determined contextually. 
insight from Kratzer that we can define a set of "best" possible worlds. ${ }^{26}$ Possibility modality, in contrast, can be conceptualized as comparison to a "lower" worldvalue that is merely contextually sufficient. It is in effect analogous to pos from the gradability literature (Kennedy 1999, among others): a proposition is a possibility if it exceeds some non-maximal contextual standard on a scale of likelihood or goodness.

Given these background assumptions, it is apparent how sets of ordered locations like (33) and sets of ordered possible worlds like (37) both share structural properties with sets of ordered degrees as in (29). All three can be modeled as sets of totally ordered abstract objects. While we will make the most of these structural similarities in our analysis of kudoka, we want to note that these domains may differ in some ways that are not relevant for our analysis. For instance, while scales of degrees and locations are dense, we don't feel that we are motivated in positing a dense scale of possible worlds. However, since density is irrelevant to our analysis, this difference (if it exists) is not an issue.

In sum, while we do not claim that all linguistic scales are formally identical, some gradable core is shared across all the domains discussed in this paper. The Logoori data in $\S 2$ shows that grammars can target this basic shared scalar structure.

\section{Analysis}

We motivate the formal ingredients of our analysis in $\S 4.1$, and provide a compositional derivation of each of the uses of kudoka in $\S 4.2$.

\subsection{Components of our analysis}

Our analysis of kudoka involves a number of different, well motivated components. In a nutshell, these are: (i) kudoka, a type-flexible two-place predicate; (ii) a contextually supplied minimum/“threshold" value that kudoka takes as its first argument; and (iii) a maximum value that kudoka takes as its second argument. Kudoka asserts that the maximum value meets or exceeds the minumum value. Kudoka additionally introduces a presupposition that both of its arguments are located on the same scale, which is necessary in order to compare them.

We locate the cross-categorial distribution of kudoka in the semantics of $k u d o k a$ itself. We treat $k u d o k a$ as type-flexible; throughout the paper, we use $\alpha$ to

26. See also Portner and Rubenstein (2016), who draw an analogy between strong necessity modals like must and "extreme" gradable adjectives like huge. 
refer to a type-flexible variable of semantic type $d, l$, or $s$. We give a semantics for kudoka in (38).

(38) $\llbracket k u d o k a \rrbracket^{c}=\lambda \alpha \lambda \alpha^{\prime} \cdot \alpha^{\prime} \geq \alpha$ defined iff

a. $\quad \alpha$ and $\alpha$ are on the same scale $\Sigma$

b. $\quad \alpha$ is of type $d$, l, or $s$ (i.e., (38) is of type $\langle d,\langle d, t\rangle\rangle,\langle l,\langle l, t\rangle\rangle$, or $\langle s,\langle s, t\rangle\rangle)$

The first argument of kudoka (of type $\alpha$ ) is the contextually supplied minimum threshold that the second argument of kudoka must meet or exceed. This threshhold argument can be realized overtly, as in Nairobi in (19), kuvunyanya imesa 'to break the table' in (13), kuromba ichai 'to make tea' in (15), and ku maragoo 'according to the law' in (21). We take this as evidence that this argument is present in the syntax.

We obtain the second argument of kudoka by movement of a constituent of type $\langle\alpha, t\rangle$. The remaining trace is of type $\alpha$ and picks out the maximum value of the moved set. Kudoka combines with this trace as its second argument. Kudoka in (38) therefore compares a maximum value to a minimum value, both of which are located on the same (totally ordered) scale, and asserts that the maximum value meets or exceeds the minumum value.

\subsection{Application of our analysis}

With these formal ingredients in hand, we derive the uses of kudoka described in $\S 2$.

\subsubsection{Degree use ('to be enough')}

We will start with what is (arguably) the most syntactically complex use of the kudoka, but also what will likely be the most familiar formally to readers: the degree use of kudoka. Degree uses of kudoka involve a relative clause. Recall from $\S 2.2$ that Logoori nominal PCLs form predicates through possessive predication, as in (7)-(8) (i.e., 'Sira is tall' $\approx$ 'Sira is with height'/'Sira has height'). In degree uses of kudoka, the nominal PCL heads a relative clause containing kudoka. We repeat a degree use of kudoka from $\S 2.2$ in (39). A literal translation of (39) could be 'The pot has heaviness that reaches breaking the table.'

(39) i-nyingu i-v-i na vu-ritu (vu-dok-an-a ku-vunyany-a 9NC-pot 9SM-COP-FV with 14NC-heaviness 14SM-DOK-PL-FV 15NC-break-FV i-mesa) 9NC-table

'The pot is heavy enough (to break the table).' 
We treat Logoori nominal PCLs as denoting portions of degree scales of type $\langle d, t\rangle$. We give a semantics for vuritu 'heaviness' in (40). Informally speaking, (40) picks out a set of degrees of heaviness. In (39), vuritu undergoes movement as the head of the relative clause, leaving behind a trace of type $d$ that picks out the maximum degree of heaviness (following the analysis of degree relatives in Grosu and Landman 1998). This maximum degree serves as the second argument of kudoka on its $\langle d,\langle d, t\rangle\rangle$ use.

(40) $\llbracket$ vuritu $\rrbracket=\lambda d$. $d$ is a degree of heaviness

We adopt an analysis of possessive predication from Francez and KoontzGarboden (2015). We locate possessive semantics in the denotation of na 'with', which is empirically motivated by the fact that $n a$ is also used in other possessive constructions in Logoori (see footnote 10). (This semantics is also proposed by Bochnak 2013, 112 for nominal PCL predications in the Bantu language Luganda.) $\mathrm{Na}$ asserts that an individual stands in a possessive relation $\pi$ with a degree of a property $P .{ }^{27}$ For more discussion of this abstract possessive relation, we refer the reader to Francez and Koontz-Garboden (2015); Bochnak (2013).

(41) a. Sira a-v-i na vu-tambe.

1Sira 1sM-COP-FV with 14NC-tallness

'Sira is tall'.

(Lit. 'Sira is with tallness.')

27. Francez and Koontz-Garboden $(2015,548)$ package both possession and context dependence in a single functional head. They model the context dependence of possessive predications like 'The pot has heaviness' $\approx$ 'The pot is heavy' through contextual domain restriction of an existential quantifier (introduced by the functional head) over portions of e.g. heaviness. For the purpose of this paper, we do not include any context dependence in the denotation of $n a$. This is because degree uses of kudoka are not context dependent. That is, (39) is felicitous even if the pot only weighs 1 pound; i.e., it would not normally count as "heavy." Correctly modeling context dependence (or lack thereof) in possessive predications is outside the scope of this paper.

Francez and Koontz-Garboden (2015) also do not use degrees to model nominal PCLs; instead, they treat nominal PCLs like strength as denoting portions of substances (analogous to the denotations of mass nouns like water, where mass nouns are modeled as ordered structures following Link 1983). Francez and Koontz-Garboden (2015, 553-554) discuss how their analysis could be recast in terms of degrees using intervals on a scale. We treat Logoori nominal PCLs as denoting sets of degrees so as to frame our proposal in terms of a familiar ordered scale, namely, the degree scale associated with gradable adjectives. This provides a good jumping-off point for introducing ordering in the spatial and modal domains. However, with additional assumptions, it is possible to recast the analysis in terms of portions. The difference is not crucial to the point being made in this paper, since degrees and portions make reference to an abstract scale. 
b. $\quad \llbracket n a \rrbracket=\lambda P_{\langle d, t\rangle} \lambda x . \exists d[P(d) \& \pi(x)(d)]$ (adapted from Francez and Koontz-Garboden 2015, 546, 548)

c. $\llbracket(41 \mathrm{a}) \rrbracket=1$ iff $\exists d[d$ is a degree of tallness $\& \pi(\operatorname{Sira})(d)]$

The first argument of kudoka on its degree use is a contextually supplied degree. This can only occur overtly as an infinitival clause, as in kuvunyanya imesa 'to break the table' in (39). We assume that this infinitival clause is nominal (Mugane 1997), and that this noun can be shifted to a degree, following arguments from Heim (1987) that nouns can denote degrees (as in amount relatives like It would take days to drink the champagne they spilled that evening). In (39), this is the degree of heaviness at which the table breaks. The degree denoted by kuvunyanya imesa in (39) is a minimum threshold value. In the absence of an overt nonfinite clause, this threshold is contextually supplied.

In (42), we give a rough semantics for the degree use of kudoka in (39). The head of the relative clause vuritu 'heaviness' combines with the predicate through Predicate Modification (Heim and Kratzer, 1998). For readability, in this and the following derivations, we do not include the presupposition from (38) that the two arguments of kudoka are on the same totally ordered scale.

(42)

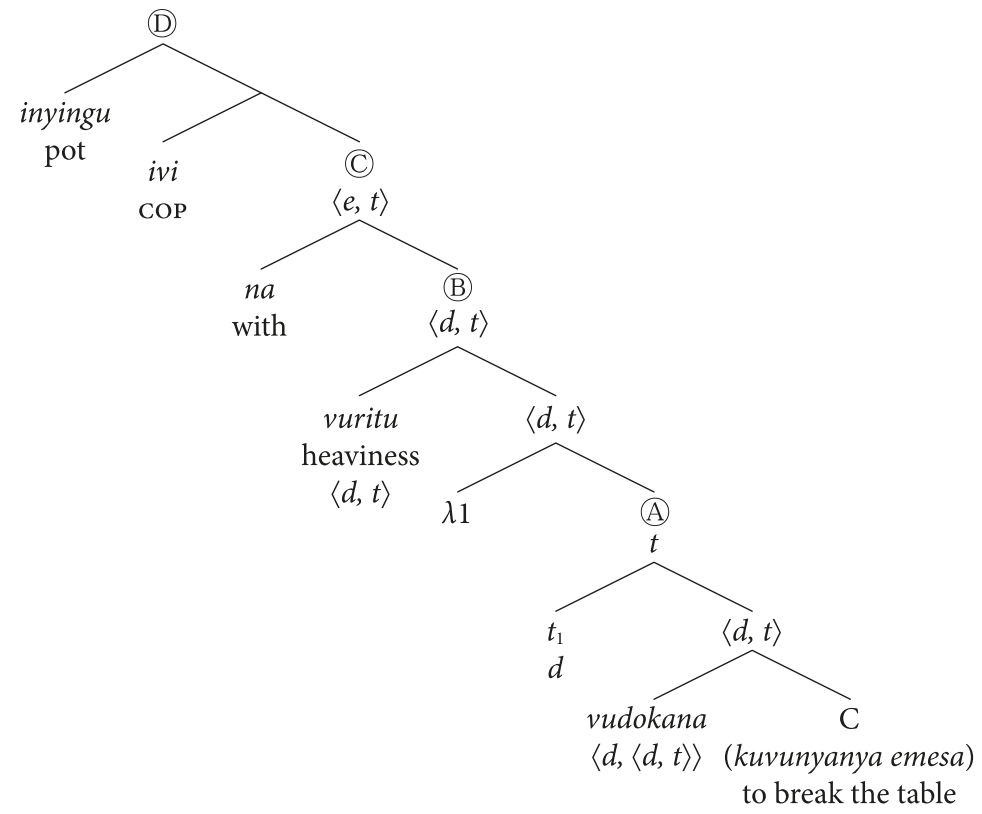

d

a. $\llbracket(A) \rrbracket^{c}=1$ iff $t_{1} \geq C_{\text {break-the-table }}$

b. $\llbracket \mathbb{B} \rrbracket^{c}=\lambda d$. $d$ is a degree of heaviness $\& d \geq \mathrm{C}_{\text {break-the-table }}$

c. $\llbracket \mathbb{C} \rrbracket^{c}=\lambda x . \exists d\left[d\right.$ is a degree of heaviness \& $\left.d \geq C_{\text {break-the-table }} \& \pi(x)(d)\right]$ 
d. $\begin{aligned} \llbracket(1) \rrbracket^{c}= & 1 \text { iff } \exists d\left[d \text { is a degree of heaviness \& } d \geq C_{\text {break-the-table }} \text { \& }\right. \\ & \pi(\operatorname{pot})(d)]\end{aligned}$

In sum, (39) is true iff the maximum degree of heaviness possessed by the pot meets or exceeds the contextually supplied minimum degree of heaviness at which the table breaks. ${ }^{28}$

\subsubsection{Amount use ('to be enough')}

Like the degree use of kudoka, the amount use of kudoka also involves a relative clause. A literal translation of (44) could be 'Sira has eggs that reach making breakfast.' We analyze the head of this relative clause as the combination of a plural count or mass noun plus some measure operator. We posit two different measure operators, CARD(INALITY) and SIZE, that combine with plural count nouns and mass nouns, respectively, and return their measures.

We begin with our analysis of the amount use of kudoka with plural count nouns, and repeat an example of this use in $(44)$ from $\S 2.3$.

(44) Sira a-v-i na ma-voyo ga-dok-an-a (ku-romb-a i-chai)

1Sira 1SM-COP-FV with 6NC-egg 6SM-DOK-PL-FV 15NC-make-FV 9NC-tea

'Sira has enough eggs (to make breakfast).'

The denotation of the cardinality function CARD is as in (45). The output of CARD applied to a plural count noun like mavoyo 'eggs' is a set of degrees that measures the cardinality of the atomic elements of the plural count noun. ${ }^{29}$

(45) $\llbracket \mathrm{CARD} \rrbracket=\lambda P_{\langle e, t\rangle} \lambda d \cdot \mu_{\text {card }}(P)=d$

where $P$ is a set of plural count individuals

We again assume syntactic movement of the head of the relative clause. In (44), the relative clause is headed by the constituent [ mavoyo CARD ] (of type $\langle d, t\rangle$ ). Its trace (of type $d$ ) picks out the maximum of the set of degrees denoted by the relative clause head; this maximum degree serves as the second argument of kudoka.

28. Our assumption about antonyms involving scale reversal (from Kennedy 2001) accounts for the interpretation of negative PCLs in kudoka-expressions. The interpretation of (43) is that the degree of the box's lightness exceeds the degree of the threshold at which it can be lifted. Lightness and weight are inversely correlated.

(43) li-boksi li-v-i na vu-angu vu-dok-an-a ku-geng-w-a 5NC-box 5SM-COP-FV with 14NC-lightness 14sM-DOK-PL-FV 15NC-lift-PASS-FV 'The box is light enough to be lifted.'

29. We assume Link (1983)'s analysis of plural count nouns as denoting sums of atomic (i.e., indivisible) elements closed under the join operation. 
The possessive predication in (44) takes the same form as the possessive PCL predication in the degree use in (39). We give a semantics for (44) in (46). (Note the structural parallels to the degree use in (42).) In short, (44) is true iff the cardinality of the set of eggs possessed by Sira meets or exceeds the contextually supplied minimum cardinality (of eggs) at which Sira can make breakfast. ${ }^{30}$

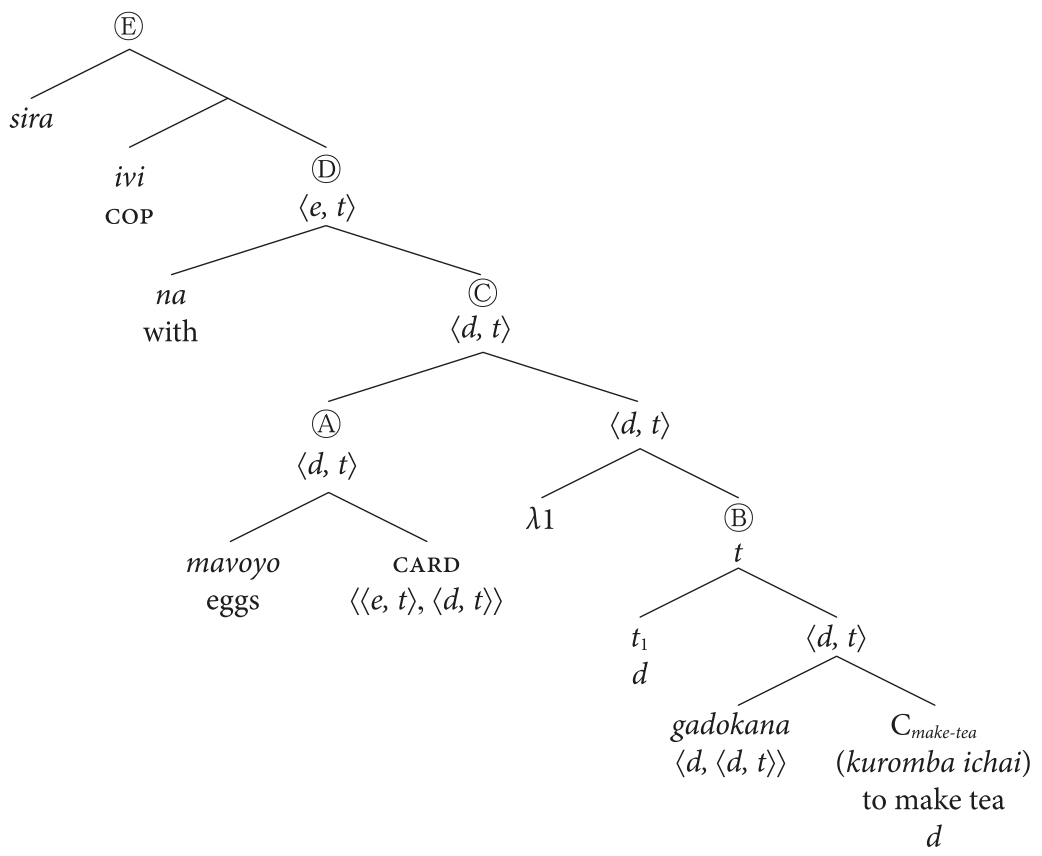

a. $\llbracket(A) \rrbracket^{c}=\lambda d \cdot \mu_{c a r d}(\lambda x \cdot \operatorname{eggs}(x))=d$

b. 【 (B) $\rrbracket^{c}=1$ iff $t_{1} \geq \mathrm{C}_{\text {make-tea }}$

c. $\llbracket$ (C) $\rrbracket^{c}=\lambda d \cdot \mu_{\text {card }}(\lambda x$. eggs $(x))=d \& d \geq \mathrm{C}_{\text {make-tea }}$

d. [ (D) $\rrbracket^{c}=\lambda x . \exists d\left[\mu_{\text {card }}(\lambda x\right.$. eggs $\left.(x))=d \& d \geq C_{\text {make-tea }} \& \pi(x)(d)\right]$

e. 【[(E) $\rrbracket^{c}=1$ iff $\exists d\left[\mu_{\text {card }}(\lambda x\right.$. eggs $\left.(x))=d \& d \geq \mathrm{C}_{\text {make-tea }} \& \pi(\operatorname{Sira})(d)\right]$

30. Here we encounter an issue that was previously discussed by Grosu and Landman (1998) in their analysis of English amount relatives (We will need the rest of our lives to drink the amount of wine they spilled that evening). Grosu and Landman (1998) argue that the amountrelative clause cannot simply denote a set of degrees, i.e. $\{d$ : they spilled $d$ that evening $\}$. The denotation of the relative clause must include what is being measured, i.e. $\{d: d$ is an amount of wine and they spilled $d$ that evening . Their solution is to propose that amount relatives are internally headed, a proposal that we also follow. In our case, we have to assume that CARD (and SIZE) output measures that also encode the kind of thing they are measuring; e.g. in (46), the scale would be one of cardinalities of sets of eggs. 
We turn next to the amount use of kudoka in combination with mass nouns. We repeat an example of this use in (47). This could be translated literally as 'We have water that reaches surviving.'
(47) ku-v-i
na a-ma-aze
ga-dok-an-i
(ku-menyel-a)
1PL-COP-FV with 6AUG-6NC-water 6SM-DOK-PL-FV 15NC-survive-FV
'We have enough water (to survive).'

We assume (following Francez and Koontz-Garboden 2015) that mass nouns like amaaze 'water' can be modeled as sets of portions of the relevant substance. ${ }^{31}$ Following Link (1983), the portions of substances that form mass nouns (like the atoms that form plural count nouns) are also ordered by the join operation. This induces a mereological part-of relation over the portions of substances, which allows the sizes of the portions to be compared..$^{32}$ These portions can be measured using a mass noun analog to CARD that maps mass individuals (i.e., sets of portions) to their sizes. (We treat mass nouns as objects of type $\langle e, t\rangle$.) We treat this measure as a degree, to bring the measures of mass nouns in line with the measures of plural count nouns. The denotation of this measure operator, sIze, is in (48).
(48)
$\llbracket$ SIZE $\rrbracket=\lambda P_{\langle e, t\rangle} \lambda d \cdot \mu_{\text {size }}(P)=d$
where $P$ is a set of portions of mass noun

We give a compositional semantics for (47) in (49). The syntax of (47) is the same as (44).

31. Francez and Koontz-Garboden (2015) treat portions as being individual-like objects of type $p$. For simplicity, we just treat them as type $e$.

32. Francez and Koontz-Garboden $(2015,553)$ further argue that the structures of mass nouns are not antisymmetric, unlike degree scales. (Given an antisymmetric ordering relation $R$, if $x R$ $y$ and $y R x$, then $x$ and $y$ occupy the same location in the ordering.) This is needed to capture the fact that two portions of a mass substance like water can be of the same size without being the same portion. However, we posit a measure operator, sIzE, that mediates between the mass noun and kudoka and outputs a degree on a scale. We claim that the measures of sizes of mass nouns can be totally ordered (and thereby antisymmetric), and don't make any claim about the structures of mass nouns themselves. 
(49)

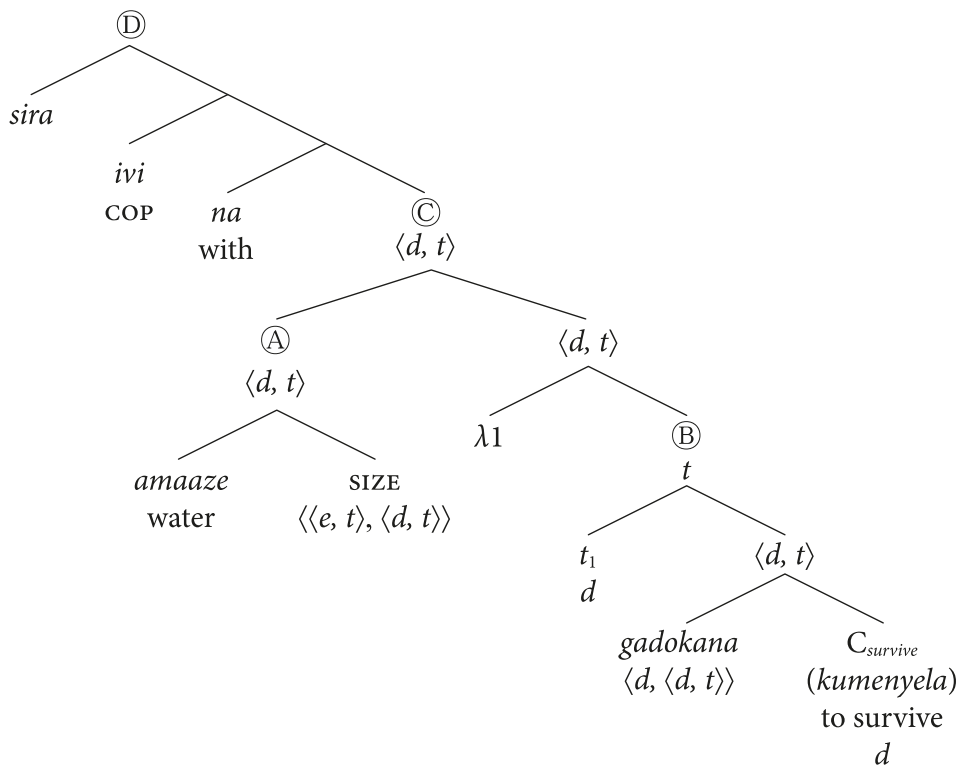

a. $\llbracket(A) \rrbracket^{c}=\lambda d \cdot \mu_{\text {size }}(\lambda x \cdot$ water $(x))=d$

b. $\llbracket(B) \rrbracket^{c}=1$ iff $t_{1} \geq \mathrm{C}_{\text {survive }}$

c. $\llbracket(C) \rrbracket^{c}=\lambda d \cdot \mu_{\text {size }}(\lambda x$. water $(x))=d \& d \geq \mathrm{C}_{\text {survive }}$

d. [D (D) $\rrbracket^{c}=1$ iff $\exists d\left[\mu_{\text {size }}(\lambda x\right.$. water $(x))=d \& d \geq \mathrm{C}_{\text {survive }} \& \pi($ we $\left.)(d)\right]$

The semantics of (47) are such that it is true iff the size of the portion of water that we possess meets or exceeds some contextually supplied minimum size (of water) that will permit us to survive. ${ }^{33}$

\subsubsection{Spatial use ('to arrive'/'to reach')}

We turn next to the spatial use of kudoka, described in $\S 2.4$ and repeated in (51). In brief, (51) asserts that the maximum location (of a set of Sira's locations with respect to Nairobi) meets or exceeds the minimum location that "counts as" being in Nairobi.

33. The intuitive meanings of both (44) and (47) seem to involve possibility modality. This was noted previously by Meier (2003) for English enough-expressions, which she proposes contain a covert conditional with a covert or overt possibility modal, like in (50).

(50) Bertha is old enough to drive a car.

$\approx$ Bertha has the age such that if Bertha is that old, she is able to drive a car

We do not think Meier (2003)'s analysis is appropriate for the Logoori data, primarily because the spatial and modal uses of kudoka do not have any intuitively conditional or possibility modal meaning. We assume that the covert modality comes from the nonfinite clause (Bhatt 1999/2006). 
(51) Sira a-dok-i (Nairobi)

1Sira 1sM-DoK-FV Nairobi

'Sira arrived (at Nairobi)'.

We begin by proposing a location function $\Lambda$ that maps an individual to the set of their locations. We give a semantics for $\Lambda$ in (52).

(52) Location function $\Lambda:^{34}$

$\llbracket \Lambda \rrbracket^{c}=\lambda x \lambda l . l$ is a location of $x$

Empirical evidence for this location function in Logoori comes from the fact that locations can be predicated directly of individuals. In (53), Sira first combines with $\Lambda$ so that the location picked out by Kenya is predicated of Sira's location, rather than the individual Sira.

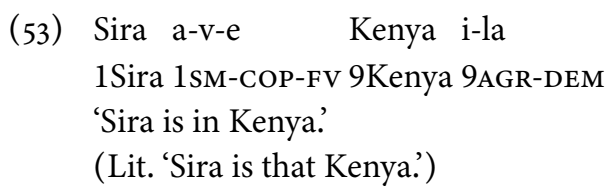

Spatial uses of kudoka like (51) necessarily involve a change in location: part of the meaning of (51) is that Sira's path began outside of Nairobi, and ended at or beyond the contextual threshold of Nairobi. We propose that this change in location stems from an presupposition that the set of locations output by $\Lambda$ is a nonsingleton set, which we omit for simplicity in our compositional semantics.

In the derivation of (51), $\Lambda$ combines with Sira to give the set of Sira's locations; following Hohaus (2020, 2018), we treat spatial paths as sets of locations. Recall from $\S 3.3$ that sets of locations are totally ordered sequentially. The output of « Sira $\Lambda \rrbracket$ is therefore a set of locations totally ordered from Sira's starting point to his end point in Nairobi. A possible path for Sira to take to Nairobi is as in Figure 2.

（54） $\llbracket \operatorname{Sira} \Lambda \rrbracket^{c}=\lambda l . l$ is a location of Sira

The constituent [ Sira $\Lambda$ ] undergoes movement to subject position. Since this set of locations is downward monotonic, the remaining trace denotes Sira's maximum location. This serves as the second argument of kudoka in its spatial use (of type $\langle l,\langle l, t\rangle\rangle)$.

The first argument of kudoka in its spatial use is also of type $l$ and can be optionally realized overtly. In (51), it is realized overtly by Nairobi. We assume that

34. The domain of $\Lambda$ is contextually restricted; it does not map an individual to all of their locations throughout their life, but to their locations within some contextually salient time interval. 


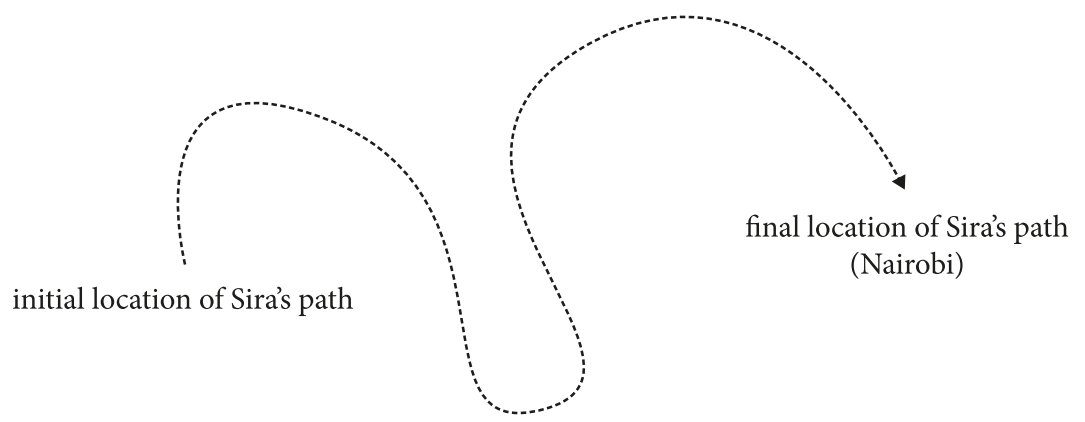

Figure 2. Graphic representation of Sira's possible path to Nairobi

the type of Nairobi here is $l$, and that this is derived by a function which maps a noun to a location, analogous to $\Lambda$ proposed above. We treat this location as a crisp threshold that varies contextually and picks out the minimum location that "counts as" being in Nairobi in the context. In some contexts, this location could be in the outskirts of the city, while in other contexts it might be the city center. We assume that this use of Nairobi to refer to something of type $l$ is derived from the individual (type $e$ ) use of Nairobi.

We give a compositional semantics for $(51)$ in $(55) .{ }^{35}$ We assume lambdaabstraction over the trace left by movement of [ Sira $\Lambda$ ] to subject. The two objects of type $\langle l, t\rangle$ combine via Predicate Modification. Existential closure binds the open location variable at the top of the tree.

(55)

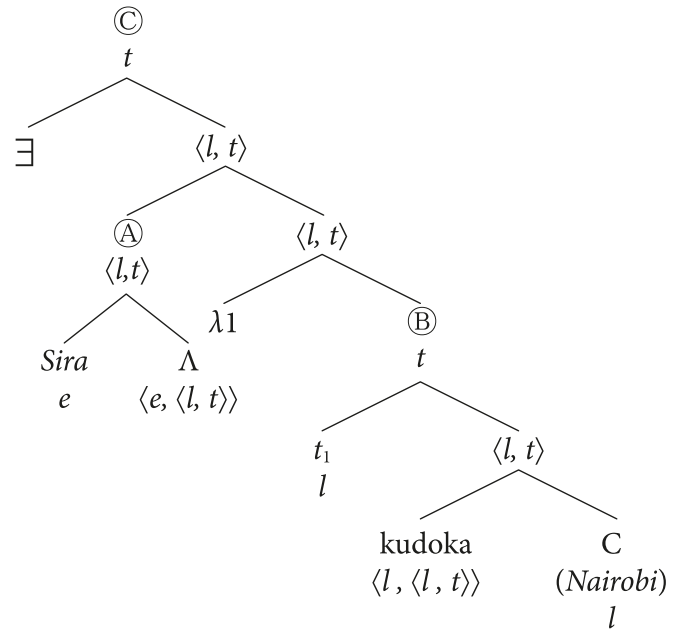

35. We put aside the morphology of kudoka in this derivation for readability. We assume that agreement morphology is associated with $\mathrm{T}$ (since it covaries with finiteness), and that verbs undergo headmovement to T. It is likely that verbs in Logoori displace further to C. 
a. $\llbracket(A) \rrbracket^{c}=\lambda l . l$ is a location of Sira

b. 【 (B) $\rrbracket^{c}=1$ iff $t_{1} \geq \mathrm{C}_{\text {Nairobi }}$

c. [I (C) $\rrbracket^{c}=1$ iff $\exists l\left[l\right.$ is a location of Sira \& $\left.l \geq \mathrm{C}_{\text {Nairobi }}\right]$

In short, (51) is true iff Sira's maximum location on a path meets or exceeds the minimum location that counts as being in Nairobi in the context. In (56), we show that it is also possible to use kudoka in a context in which Sira reached Nairobi and then passed beyond and out of Nairobi, as predicted by the use of the $\geq$ relation in our semantics in (55).

(56) Sira a-duk-i Nairobi, kare ndio a-l-i Kisumu

1Sira 1sM-DOK-FV 9Nairobi, in.fact 1sM-COP-FV 9Kisumu

'Sira reached Nairobi, in fact, he's in Kisumu.'

\subsubsection{Modal use ('must')}

Finally, we discuss the modal use of kudoka, in which it contributes necessity modality.

Recall from $\$ 3.4$ that modals can be modeled using totally ordered scales of possible worlds (Lassiter 2014, 2017b). Since worlds are not inherently totally ordered, we propose that in Logoori, this total ordering is imposed by the subjunctive mood. Subjunctive is required in the presence of kudoka, and is marked by the verbal suffix -e.

This proposal is in the spirit of Villalta (2008)'s observation that there is a link between subjunctive mood and gradability. ${ }^{36}$ We give a semantics for the subjunctive in (57); the dimension of the scale $\Sigma$ is contextually supplied and can be e.g. goodness with respect to the law (deontic modality), likelihood (epistemic modality), et cetera.

$\llbracket \operatorname{SBJV} \rrbracket^{c}=\lambda p_{\langle s, t\rangle} \lambda w \cdot \operatorname{VALUE}_{\Sigma, c}(p)=w$

36. We follow the ideas concerning subjunctive mood introduced in Villalta (2008) in spirit, but not formalism. In Villalta's approach, gradability is attributed to the predicates that embed a subjunctive complement. On our approach, the subjunctive introduces an ordering, and the embedding predicate (here, kudoka) establishes a "comparison."

We do not posit a measure function here because we do not wish to map the sets of worlds to the set of real numbers (or to a set of degrees). This is because it is not clear whether the ordering imposed on worlds shares the properties of real numbers/degree scales in being (a) downward monotonic, and (b) dense. For our analysis to work, we must minimally assume that the ordering is downward monotonic, because we invoke maximality. However, we do not necessarily need the ordering to be dense. We refer the reader to the discussions in Lassiter (2017a); Portner and Rubenstein (2016). 
The function value requires some more explanation. Propositions, like worlds, are not inherently ordered; they are simply the set of worlds in which $p$ is true. value allows us to translate from propositions to ordered worlds. This in turn lets us compare the values of propositions, which can be reconstructed from these ordered worlds. We crucially adopt Lewis' view of comparing propositions in that "we are comparing $\varphi$-at-its-best with $\psi$-at-its-best, and ignoring the non-best ways for $\varphi$ and $\psi$ to hold" (Lewis, 1973, 101) cited in Lassiter to appear, 13. This is, in Lewis' terms, "maximax betterness." We use the best world in the set of $p$-worlds to globally assign a value to $p \cdot{ }^{37}$ This is how value maps propositions to ordered worlds (we leave open the precise mathematical calculation involved). Given our assumption of Lewis's maximax betterness, we can then reconstruct orderings of propositions from these ordered worlds.

Given some ordered scale of worlds, we can delimit which worlds are the most ideal along some parameter - essentially adopting the core insights of Lewis (1973) and Kratzer $(1981,1991)$. In this model, there is some $w$ on the scale such that this world meets the minimum number of requirements to be considered "ideal." Because the scale is a total ordering, any world above $w$ is at least as good as $w$ - in fact, better. In this way, we can delimit the set of best possible worlds.

If the value of a proposition $p$ (i.e., the value of the best $p$-world) meets or exceeds the value of the sufficiently ideal world, then $p$ is a necessity. Reworded: a proposition $p$ is a necessity if the best $p$-world is at least as good as the least best world on a given modal scale. Alternatively: if ideal worlds are $p$-worlds, then $p$ is a necessity. (As we discussed in $\S 3.4$, possibility modality is derived the same way, but with a lower threshhold.) We represent this model of necessity modality graphically in (58). Note that this parallels our models for the spatial and degree uses of kudoka in that it also refers to a scale with a threshold value that is met or exceeded.

37. In the theory developed in Lassiter (2010) et seq, Lassiter fundamentally rejects the gradable semantics that Lewis et seq assumes, and in particular rejects maximax. In Lassiter's approach, a proposition's overall goodness can be calculated by taking the weighted average of the worlds in $p$. (The weight of each world is a function of how probable that world is if $p$ is true.) It is also possible to re-frame our proposal along these lines, though it requires a number of additional assumptions and mathematical processes. It is important to recognized that maximax and (weighted) averaging are both ways to "lift" world-ranking to proposition-ranking; we refer the reader to the discussion in Lassiter (to appear) on issues with these various theories. 
(58)

value of p's

goodness

scale of ranked

world-values

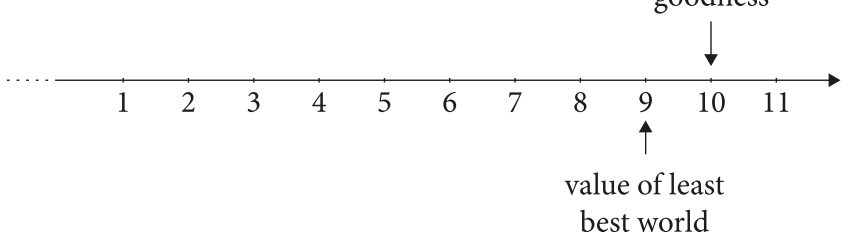

In our compositional analysis of modal uses of kudoka, we treat the least-best threshold value as something of type $s$ that is supplied contextually and that serves as the first argument of $k u d o k a$ (in its $\langle s,\langle s, t\rangle\rangle$ use). It can optionally occur overtly; in (59) (repeated from $\S 2.5$ ), it occurs overtly as ku maragoo 'according to the law.' We can think of this threshold as the least best world in which the law is obeyed.

(59) (ku ma-ragoo) ku-dok-a Sira a-zi-e yeng'o

to 6NC-law 15NC-DoK-Fv 1Sira 1sm-go-sBjv home

'(According to the law), Sira must go home.'

In (59), the constituent [ Sira azie yeng'o ] undergoes extraposition to the right edge of the clause. This moved constituent of type $\langle s, t\rangle$ leaves behind a trace of type $s$, which serves as the second argument of kudoka. Because we assume that the ordering imposed by SBJV is downward monotonic, this trace picks out the highest ranked world in which Sira goes home on a scale of law-abiding-ness. Kudoka contributes that this world meets or exceeds the contextually supplied least-best world according to the law.

We assume lambda-abstraction over the trace, and combination of the two objects of type $\langle s, t\rangle$ via Predicate Modification. The open world variable at the top of the tree is bound by existential closure.

$(60)$
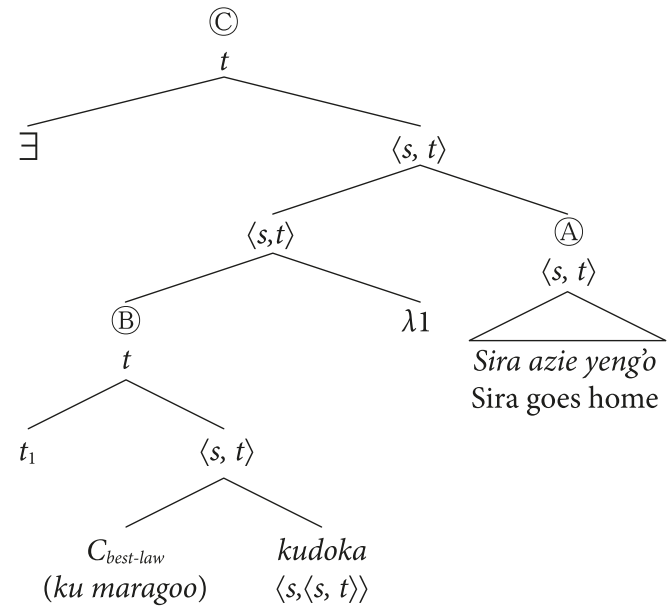

according to the law 
a. $\llbracket(A) \rrbracket^{c}=\lambda w^{\prime}$. value $\sum, c\left(\lambda w^{\prime}\right.$. Sira goes home in $\left.w^{\prime}\right)=w$

b. $\llbracket(B) \rrbracket^{c}=1$ iff $t_{1} \geq \mathrm{C}_{\text {best-law }}$

c. $\llbracket\left(C \rrbracket^{c}=1\right.$ iff $\exists w \operatorname{valuE}_{\Sigma, c}\left(\lambda w^{\prime}\right.$ Sira goes home in $\left.\left.w^{\prime}\right)=w \& w \geq \mathrm{C}_{\text {best-law }}\right]$

(59) is true iff there exists a world in which Sira goes home, and the value of this world on a scale of law-abiding-ness meets or exceeds the value of the least best possible world on a scale of law-abiding-ness. That is, (59) is true iff the proposition "Sira goes home" is a necessity according to the law.

Though the truth conditions in (59) involve existential quantification over worlds, recall that the notions of existential and universal quantification are not applicable to a gradable treatment of modality; see discussion in $\S 3.4$.

\subsection{Recap}

To summarize, all of the uses of kudoka in $\S 2$ can be modeled using the same basic, type-flexible semantics. In all of its uses, it contributes a $\geq$ relation between its two arguments, where its first argument is a contextually supplied threshold value and its second argument is a maximal value denoted by a trace. To be able to compare them, kudoka presupposes that both of these values are located on the same (totally ordered) scale.

Although the denotation of kudoka is consistent across all uses, it appears in different syntactic configurations. In its spatial use, kudoka occurs as a main predicate; in its degree and amount uses, it occurs in a relative clause modifying a noun; in its modal use, it is syntactically peripheral. We account for its crosscategorial distribution by treating it as type-flexible.

\section{An alternate analysis: Shifting to degrees}

One of the major issues raised by the data in $\S 2$ is how to account for the crosscategorial distribution of kudoka. We take this cross-categorial distribution to reflect semantic type-flexibility at some point in the composition. We have posited that kudoka is type-flexible, but an alternative is to propose that (i) type-flexibility is implemented via a type-flexible measure function; and (ii) kudoka is typeinflexible and relates two degree arguments. In this section, we briefly review this idea, which we call the "degree analysis", and explain why we believe that it is inferior to the proposal we give in $\S 4$.

The motivation behind the degree analysis is to make the most of the extensive prior literature on degree scales (Cresswell 1976, Kennedy 1999, among many others). As we discussed in $\$$ 3.1, degree scales are canonically assumed in the lit- 
erature on gradable predicates to be totally ordered. Mapping worlds onto degree scales would therefore accomplish what we currently achieve with the ordering presupposition introduced by the subjunctive.

Shifting types to degrees can be accomplished by the type-flexible measure function $\mu \Sigma$ in (61), where $\Sigma$ refers to the contextually supplied dimension of the scale. This already presupposes a scalar structure for sets of locations and possible worlds and assumes that these scales are downward monotonic, and that $\mu \Sigma$ therefore picks out their maximal world/location, which could be converted to a degree. ${ }^{38}$ The measure function is of type $\langle\langle l, t\rangle, d\rangle$ or $\langle\langle s, t\rangle, d\rangle$; its output serves as the second argument of kudoka.

(61) Measure function:

$\llbracket \mu \Sigma \rrbracket^{c}=\lambda \mathrm{A}_{\langle\alpha, t\rangle}$. degree of A on a scale $\Sigma$

where $\mathrm{A}$ is of type $\langle l, t\rangle$ or $\langle s, t\rangle$

Under this analysis, kudoka asserts that its second argument meets or exceeds its first argument, where both arguments are of type $d$. This is identical to its denotation in (38), modulo its type-inflexibility.

(62) $\llbracket k u d o k a \rrbracket^{c}=\lambda \mathrm{C}_{d} \lambda \mathrm{d} . \mathrm{d} \geq \mathrm{C}$

where $\mathrm{C}$ is a contextually supplied threshold degree

Conceptually, we feel that this degree analysis misses a larger generalization that can be made about cross-categorial gradability in grammar. Sets of locations and possible worlds are translatable into degrees via $\mu_{\Sigma}$ because they share properties with sets of degrees: they can also be modeled as ordered objects on a scale. Although degrees constitute the most familiar scales in the literature, shifting everything into degrees obscures the fact that gradability also exists in other domains. We chose to discard $\mu_{\Sigma}$ and model $k u$ doka as type-flexible for this reason.

Furthermore, we feel that the degree analysis rests too heavily on the measure function in (61). In the degree analysis, $\mu_{\Sigma}$ serves two functions: (i) mapping sets of locations or worlds to a contextually specified, totally ordered (degree) scale $\Sigma$, and (ii) picking out the maximum degree on that scale. $\mu_{\Sigma}$ therefore both type-shifts and enforces a total ordering, whereas kudoka in (38) simply presupposes that both of its arguments are located on the same scale. Given a downward monotonic scale, the syntactically motivated movement operations described in $\S 4$ accomplish the same result as the maximality function of $\mu_{\Sigma}$. We can therefore

38. In our current analysis, maximality is achieved by composition with a trace left by movement. In the degree analysis, no movement is necessary for semantic composition except in the relative clauses (degree and amount uses), in which case $\mu_{\Sigma}$ becomes unnecessary. 
unpack the contribution of $\mu_{\Sigma}$ into two simpler components: the scale presupposition of kudoka and movement in the syntax.

Finally, shifting everything to degrees predicts that modality and space have all the properties associated with degrees, including density and monotonicity. This is unlikely, for the reasons discussed earlier. The point underscores what we feel is the core takeaway from the data above. PCLs, modal expressions, and spatial expressions all share a core kernel of meaning, but they are distinct domains which cannot - and should not - be entirely conflated. We leave it for future research along which parameters the three domains differ.

\section{Conclusion}

Linguistic expressions referring to spatial paths, gradable predicates, measures of plural count nouns/mass nouns, and modals can be treated similarly in the grammar. We demonstrated this using data from Logoori kudoka, which displays a "polysemy" that spans the relevant domains.

As noted in $\S 1$, many other languages share this polysemy. We conclude by noting some additional language internal evidence for a shared meaning. Other constructions in Logoori also conflate space and degrees; for instance, Logoori uses the verb kuveta 'to pass' in comparisons (this relation between spatial and comparative meaning has been described by typologists in many other languages; Stassen 1985).

(63) a. li-gaara li-vet-ang-a

5NC-train 5sM-pass-PROG-FV

'The train is passing.'

b. Sira a-v-i na vu-yanzi ku-vet-a Imali

1Sira 1sM-COP-GV with 14NC-happiness 15NC-pass-FV 1Imali

'Sira is happier than Imali.'

Logoori also uses the deictic pronoun ho 'there' in comparative constructions in which the standard is implicit (note the lack of kuveta 'to pass,' or any other comparative morphology). This puzzling data again demonstrates a relationship between spatial expressions and gradable predicates.

(64) a. lu-heni lu-lasan-i ho

11NC-lightning 11sm-flash-Fv there

'Lightning flashed there.' 


\section{b. Sira ni mu-gera ho \\ 1Sira COP 1AGR-smart there \\ 'Sira is smarter'.}

This study has implications for semantic change and the diachronic source of threshhold expressions like kudoka. Although we can only speculate at present, our hypotheses are supported by data from other languages in the Luhia subfamily of Bantu. A number of other Luhya languages have similarly polysemous lexical items. In (65), we list the relevant lexical items on the left, and their respective languages in bold on the right..$^{39}$ All of these lexical items can express spatial and modal uses akin to kudoka; degree and amount uses are available for a subset of these. (Of these lexical items, only Lutiriki khutukha 'to arrive'/'must' is clearly cognate with Logoori $k u d o k a .^{40}$ This again shows that this polysemy is not unique to kudoka, and that there is some underlying semantic relationship between the spatial, degree, amount, and modal meanings.)

(65) 'to arrive' / 'must'
a. kwenyekha, khoya
b. okhwola
c. kukhoyera
d. khutukha
e. okhula

\section{Lubukusu \\ Lunyore \\ Lusaamia \\ Lutiriki \\ Luwanga}

All of the Luhia lexical items that we have collected so far (as well as the crosslinguistic examples in $\S 1$ ) display the spatial use: we have not found any lexical item that displays only the degree/amount and modal uses. This suggests to us that the spatial use of these items is basic, and that the degree/amount and modal uses arose out of their spatial meaning. This accords with Hohaus (2020)'s proposal for a reanalysis of spatial paths into degree scales in Samoan.

Given a lexical item with spatial, degree/amount, and modal uses (like Logoori kudoka or Mandarin Chinese gou), it is not possible at present to determine whether the degree/amount or modal meaning arose first out of the spatial meaning. Alternately, the two meanings could have arisen simultaneously. Different languages could also have developed different meanings from the spatial use. For instance, the lexical item okhwola (which is not cognate with Logoori's kudoka) in the Luhia language Nyala East shows only the spatial and degree/ amount uses, not the modal uses. Nyala East okhwola could have developed the degree use first, while other Luhia languages developed the modal use first. In

39. This data is from the second author's fieldwork with speakers of Luhia languages in Kenya. 40. Lubukusu kwenyekha is cognate with Logoori kwenya; in Logoori, this lexical item expresses 'to want'/'should' (Gluckman et al. 2017). 
any case, this proposal suggests a novel "map" for the development of modal meanings, of the kind discussed by Bybee et al. (1994) and van der Auwera and Plungian (1998). To our knowledge, this change from spatial meaning $\rightarrow$ degree meaning $\rightarrow$ modal meaning is a novel observation.

We note that our analysis does not predict the strength of the resulting modal, i.e., whether it will be necessity or possibility. As we described in $\S 4.2 .4$, under a gradable analysis of modality, all that is required for a possibility modal is that the proposition's value meet or exceed a contextually specified point on the scale. For necessity modality, the point is high on the scale, while for possibility it is relatively low. Thus, the fact that Logoori kudoka marks necessity while Mandarin Chinese gou marks possibility (2c) is not an issue for our proposal.

We will close with a brief discussion of further extensions of our proposal. Our current analysis of kudoka is one in which it is restricted to combining with arguments of certain semantic types, namely, $d, l$, or $s$. However, one could ask whether it is able to combine with arguments of other semantic types, as long as those arguments denote values on a scale. For example, could kudoka combine with time arguments of type $i$, located on ordered scales of times? We may also ask whether events can be modified by kudoka. Preliminary data like (66) suggests that this is possible, in which the relevant scale in (66) could be the intensity of Sira's running. However, speakers are much more likely to use sam (discussed in footnote 11) to express such meanings.

li-nagura li-a Sira li-dok-an-i ku-guta ma-shindano.
5NC-run 5AGR-ASso 1Sira 5AGR-DOK-PL-FV 15NC-winfV 6NC-race
'Sira's running was enough to win the race.'

In sum, we have shown in this paper that spatial expressions, gradable predicates, measures of amounts, and modals can be treated similarly in the grammar, as demonstrated by the Logoori verb kudoka. It is an open question as to why in some languages, only subsets of these meanings arise. We hypothesize that at least some of the explanation resides in lexical category and c-selectional restrictions. We speculate that the degree ('to be enough') use of kudoka arose because (some) PCLs in Logoori are nouns, and so can act as subjects of the verb. The modal use then likely developed later as the meaning of kudoka further bleached and cselectional requirements were relaxed.

\section{Funding}

This work has been supported in part by European Research Council Consolidator Grant ERC$2017-$ COG 769192. 


\section{Acknowledgements}

All of the Logoori data in this paper is from our own fieldwork (with Mwabeni Indire, Walter Kigali, Bernard Chahilu, and Ben Lavussa). Thank you to our wonderful consultants! We would also like to thank Vera Hohaus, Andrew Koontz-Garboden, Ryan Bochnak, Emily Hanink; audience members at Triple A 6, SALT 30, and the University of Manchester's Semantics Lab; and our anonymous Linguistic Variation, Triple A 6 and SALT 30 reviewers for their helpful comments.

\section{Abbreviations}

We use the following abbreviations:

$\begin{array}{llll}1 / 2 / 3 \ldots & \text { noun classes } & \text { MOD } & \text { modal element } \\ \text { AC } & \text { anticausative } & \text { NEG } & \text { negative } \\ \text { CAUSE } & \text { causative } & \text { PASS } & \text { passive } \\ \text { COMP } & \text { complementizer } & \text { REC } & \text { reciprocal } \\ \text { COP } & \text { copula } & \text { SBJV } & \text { subjunctive } \\ \text { FUT } & \text { future } & \text { SG/PL } & \text { singular/plural } \\ \text { FV } & \text { final vowel } & \text { SM } & \text { subject marker }\end{array}$

Throughout the paper, we gloss the verb root of kudoka as DoK. We assume the standard Logoori orthography; $\left\langle n g^{\prime}\right\rangle$ represents a phonemic velar nasal $/ \mathrm{y} /$ and $\langle n y\rangle$ represents a phonemic palatal nasal /n/. Logoori has a two-tone system (Samuels and Paster 2015), which is not represented in this orthography.

\section{References}

Bartsch, R. and Vennemann, T. (1972). The grammar of relative adjectives and comparison. Linguistische Berichte, 21:168-185.

Beck, S., Krasikova, S., Fleischer, D., Gergel, R., Hofstetter, S., Savelsberg, C., Vanderelst, J., and Villalta, E. (2009). Crosslinguistic variation in comparison constructions. Linguistic Variation Yearbook 2009, 9:1-66. https://doi.org/10.1075/livy.9.01bec

Bhatt, R. (1999). Covert Modality in Non-finite Contexts. PhD thesis, University of Pennsylvania.

Bhatt, R. (2006). Covert Modality in Non-finite Contexts. Mouton de Gruyter. https://doi.org/10.1515/9783110197341

Bierwisch, M. (1989). The semantics of gradation. In Bierwisch, M. and Lang, E., editors, Dimensional Adjectives: Grammatical Structure and Conceptual Interpretation, pages 71-261. Springer Verlag. https://doi.org/10.1007/978-3-642-74351-1_3

Bochnak, R. (2013). Cross-linguistic Variation in the Semantics of Comparatives. PhD thesis, University of Chicago.

Bostoen, K., Dom, S., and Segerer, G. (2015). The antipassive in Bantu. Linguistics, 53(4):731-772. https://doi.org/10.1515/ling-2015-0016 
Bowler, M. and Gluckman, J. (2020). Cross-categorial gradability in Logoori. In Proceedings of Semantics and Linguistic Theory 30.

Bybee, J., Perkins, R., and Pagliuca, W. (1994). The Evolution of Grammar: Tense, Aspect, and Modality in the Languages of the World. University of Chicago Press.

Cresswell, M. (1976). The semantics of degree. In Partee, B., editor, Montague Grammar, pages 261-292. Academic Press, New York. https://doi.org/10.1016/B978-0-12-545850-4.50015-7

Cresswell, M. (1978). Prepositions and points of view. Linguistics and Philosophy, 2(1):1-41. https://doi.org/10.1007/BFo0365129

Dixon, R. (1982). Where Have All the Adjectives Gone? And Other Essays in Semantics and Syntax. Mouton, The Hague. https://doi.org/10.1515/9783110822939

Dom, S., Kulikov, L., and Bostoen, K. (2018). Valency-decreasing derivations and quasimiddles in Bantu: A typological perspective. Southern African Linguistics and Applied Language Studies, 36(3):165-173. https://doi.org/10.2989/16073614.2018.1552164

Faller, M. (1999). Dimensional adjectives and measure phrases in vector space semantics. In Martina Faller, S. K. and Pauly, M., editors, Formalizing the Dynamics of Information, pages 151-170. CSLI Publications.

Francez, I. and Koontz-Garboden, A. (2015). Semantic variation and the grammar of property concepts. Language, 91(3):533-563. https://doi.org/10.1353/lan.2015.0047

Francez, I. and Koontz-Garboden, A. (2017). Semantics and Morphosyntactic Variation: Qualities and Grammar of Property Concepts. Oxford University Press. https://doi.org/10.1093/acprof:oso/9780198744580.001.0001

Gluckman, J. (2018). Iterative-reciprocal polysemy in Logoori. In Bochnak, R., editor, Proceedings of The Semantics of African, Asian and Austronesian Languages 6.

Gluckman, J. and Bowler, M. (2016). Expletive agreement, evidentiality, and modality in Logooli. In Proceedings of Semantics and Linguistic Theory 26, pages 1063-1082. https://doi.org/10.3765/salt.v26io.3935

Gluckman, J. and Bowler, M. (2020). The expression of modality in Logoori. Forthcoming in Journal of African Languages and Linguistics. https://doi.org/10.1515/jall-2020-2010

Gluckman, J., Bowler, M., Sifuna, M., Alulu, K., and Diercks, M. (2017). A typological study of modality in Luhya languages. Handout, Annual Conference on African Linguistics 48.

Grosu, A. and Landman, F. (1998). Strange relatives of the third kind. Natural Language Semantics, 6:125-170. https://doi.org/10.1023/A:1008268401837

Halpert, C. (To appear). The augment. In The Oxford Guide of Bantu Languages. Oxford University Press.

Heim, I. (1987). Where does the definiteness restriction apply? Evidence from the definiteness of variables. In Ter Meulen, A. and Reuland, E., editors, The Representation of (In)definiteness, pages 21-42. MIT Press, Cambridge.

Heim, I. and Kratzer, A. (1998). Semantics in Generative Grammar. Blackwell Publishers.

Hohaus, V. (2012). Directed motion as comparison: Evidence from Samoan. In Bogal-Allbritten, E., editor, Proceedings of SULA 6, pages 335-348.

Hohaus, V. (2018). How do degrees enter the grammar? Language change in Samoan from [-DSP] to [+DSP]. In Bogal-Allbritten, E. and Coppock, E., editors, Proceedings of The Semantics of African, Asian and Austronesian Languages 4, pages 106-120.

Hohaus, V. (2020). Language change and the Degree Semantics Parameter. Manuscript. Kennedy, C. (1999). Projecting the Adjective: The Syntax and Semantics of Gradability and Comparison. Garland, New York. 
Kennedy, C. (2001). Polar opposition and the ontology of 'degrees'. Linguistics and Philosophy, 24(1):33-70. https://doi.org/10.1023/A:1005668525906

Kennedy, C. (2011). Vagueness and comparison. In Egré, P. and Klinedinst, N., editors, Vagueness and Language Use, pages 73-97. Palgrave Macmillan, London. https://doi.org/10.1057/9780230299313_4

Kennedy, C. and McNally, L. (2005). Scale structure and semantic typology of gradable predicates. Language, 81(2):345-81. https://doi.org/10.1353/lan.2005.0071

Klecha, P. (2014). Bridging the Divide: Scalarity and Modality. PhD thesis, University of Chicago.

Kratzer, A. (1981). The notional category of modality. In Eikmeye, H. and Rieser, H., editors, Words, Worlds, and Contexts: New Approaches in Word Semantics, pages 38-74. Mouton de Gruyter, Berlin. https://doi.org/10.1515/9783110842524-004

Kratzer, A. (1991). Modality. In von Stechow, A. and Wunderlich, D., editors, Semantics: An International Handbook of Contemporary Research, pages 639-650. Mouton de Gruyter.

Krifka, M. (1998). The origins of telicity. In Events and Grammar, pages 197-235. Springer, Dordrecht. https://doi.org/10.1007/978-94-011-3969-4_9

Lassiter, D. (2010). Gradable epistemic modals, probability, and scale structure. In Proceedings of Semantics and Linguistic Theory 20.

Lassiter, D. (2011). Measurement and modality. PhD thesis, New York University.

Lassiter, D. (2014). Modality, scale structure, and scalar reasoning. Pacific Philosophical Quarterly, 95:461-49o. https://doi.org/10.1111/papq.12045

Lassiter, D. (2017a). Graded modality. Manuscript, Stanford University.

Lassiter, D. (2017b). Graded Modality: Qualitative and Quantitative Perspectives. Oxford University Press. https://doi.org/10.1093/oso/9780198701347.001.0001

Lassiter, D. (in press). Graded modality. In Blackwell Companion to Semantics. WileyBlackwell. https://doi.org/10.1093/oso/9780198701347.001.0001

Lewis, D. (1973). Counterfactuals. Harvard University Press.

Link, G. (1983). The logical analysis of plurals and mass terms: A lattice-theoretic approach. In Portner, P. and Partee, B., editors, Formal Semantics: The Essential Readings, pages 127-146. Blackwell.

Maslova, E. (2003). Tundra Yukaghir, volume 372 of Languages of the World. LINCOM Europa, Munich.

Meier, C. (2003). The meaning of too, enough, and so... that. Natural Language Semantics, 11:69-107. https://doi.org/10.1023/A:1023002608785

Mugane, J. (1997). Bantu Nominalization Structures. PhD thesis, University of Arizona.

Munro, P. and Willmond, C. (1995). Chickasaw: An Analytical Dictionary. University of Oklahoma Press, Norman.

Nurse, D. and Philippson, G. (2003). The Bantu Languages. Routledge.

Portner, P. (2009). Modality. Oxford University Press.

Portner, P. and Rubenstein, A. (2016). Extreme and non-extreme deontic modals. In Charlow, N. and Chrisman, M., editors, Deontic Modality. Oxford University Press. https://doi.org/10.1093/acprof:oso/9780198717928.003.0010

Rett, J. (2014). The polysemy of measurement. Lingua, 143:242-266.

Rett, J. (2015). The Semantics of Evaluativity. Oxford University Press.

Rett, J. (2018). The semantics of many, much, few, and little. Language and Linguistics Compass, 12:1-18. https://doi.org/10.1111/Inc3.12269 
Samuels, A. and Paster, M. (2015). Verbal tone in Logoori. Handout from Workshop on Luyia Bantu Languages at Annual Conference on African Linguistics 46.

Schwarzschild, R. (2012). Directed scale segments. In Proceedings of Semantics and Linguistic Theory 22, pages 65-82. https://doi.org/10.3765/salt.v22io.2634

Schwarzschild, R. (2013). Degrees and segments. In Proceedings of Semantics and Linguistic Theory 23, pages 212-238. https://doi.org/10.3765/salt.v23io.2661

Seidl, A. and Dimitriadis, A. (2003). Statives and reciprocal morphology in Swahili. Typologie des langues d'Afrique et universaux de la grammaire.

Stassen, L. (1985). Comparison and Universal Grammar. Basil Blackwell.

van der Auwera, J. and Plungian, V. (1998). Modality's semantic map. Linguistic Typology, 2:79-124. https://doi.org/10.1515/lity.1998.2.1.79

Vander Klok, J. (2014). Questionnaire on modality for cross-linguistic use. http://www.eva .mpg.de/lingua/tools-at-lingboard/questionnaire/cross-linguisticuse.php

Villalta, E. (2008). Mood and gradability: an investigation of the subjunctive mood in Spanish. Linguistics and Philosophy, 31:467-522. https://doi.org/10.1007/s10988-008-9046-x

von Stechow, A. (1984). My reaction to Cresswell's, Hellan's, Hoeksema's and Seuren's comments. Journal of Semantics, 3:183-199. https://doi.org/10.1093/jos/3.1-2.183

Winter, Y. (2005). Cross-categorial restrictions on measure phrase modification. Linguistics and Philosophy, 28(2):233-267. https://doi.org/10.1007/s10988-004-1469-4

Yalcin, S. (2010). Probability operators. Philosophy Compass, pages 916-937. https://doi.org/10.1111/j.1747-9991.2010.00360.x

\title{
Address for correspondence
}

\author{
Margit Bowler \\ Department of Linguistics and English Language \\ University of Manchester \\ Oxford Road \\ Manchester M13 9PL \\ United Kingdom \\ margitbowler@gmail.com
}

\section{Co-author information}

John Gluckman

Department of Linguistics

University of Kansas

johngluckman@gmail.com 


\section{Publication history}

Published online: 18 January 2021

Corrected: 9 June 2021

In the original Online-First version of this article published on 18 January 2021, the funding details were incomplete. These have been updated in the current version of the article. 